\title{
A Machine Learning Approach to Optimal Tikhonov Regularization I: Affine Manifolds
}

\author{
Ernesto De Vito (corresponding author) \\ DIMA, Università di Genova, Via Dodecaneso 35, Genova, Italy \\ DEVITO@DIMA.UNIGE.IT \\ Massimo Fornasier \\ MASSIMO.FORNASIER@MA.TUM.DE \\ Technische Universität München, Fakultät Mathematik, Boltzmannstrasse 3 D-85748, \\ Garching bei München, Germany
}

Valeriya Naumova

VALERIYA@SIMULA.NO

Simula Research Laboratory, Martin Linges vei 25, Fornebu, Norway

\begin{abstract}
Despite a variety of available techniques the issue of the proper regularization parameter choice for inverse problems still remains one of the relevant challenges in the field. The main difficulty lies in constructing a rule, allowing to compute the parameter from given noisy data without relying either on any a priori knowledge of the solution or on the noise level. In this paper we propose a novel method based on supervised machine learning to approximate the high-dimensional function, mapping noisy data into a good approximation to the optimal Tikhonov regularization parameter. Our assumptions are that solutions of the inverse problem are statistically distributed in a concentrated manner on (lowerdimensional) linear subspaces and the noise is sub-gaussian. We show that the number of previously observed examples for the supervised learning of the optimal parameter mapping scales at most linearly with the dimension of the solution subspace. Then we also provide explicit error bounds on the accuracy of the approximated parameter and the corresponding regularization solution. Even though the results are more of theoretical nature, we present a recipe for the practical implementation of the approach, we discuss its computational complexity, and provide numerical experiments confirming the theoretical results. We also outline interesting directions for future research with some preliminary results, confirming their feasibility.
\end{abstract}

Keywords: Tikhonov regularization, parameter choice rule, sub-gaussian vectors, high dimensional function approximations, concentration inequalities.

\section{Introduction}

In many practical problems, one cannot observe directly the quantities of most interest; instead their values have to be inferred from their effects on observable quantities. When this relationship between observable $Y$ and the quantity of interest $X$ is (approximately) linear, as it is in surprisingly many cases, the situation can be modeled mathematically by the equation

$$
Y=A X
$$

for $A$ being a linear operator model. If $A$ is a "nice", easily invertible operator, and if the data $Y$ are noiseless and complete, then finding $X$ is a trivial task. Often, however, the 
mapping $A$ is ill-conditioned or not invertible. Moreover, typically (1) is only an idealized version, which completely neglects any presence of noise or disturbances; a more accurate model is

$$
Y=A X+\eta,
$$

in which the data are corrupted by an (unknown) noise. In order to deal with this type of reconstruction problem a regularization mechanism is required (Engl et al., 1996).

Regularization techniques attempt to incorporate as much as possible an (often vague) a priori knowledge on the nature of the solution $X$. A well-known assumption which is often used to regularize inverse problems is that the solution belongs to some ball of a suitable Banach space.

Regularization theory has shown to play its major role for solving infinite dimensional inverse problems. In this paper, however, we consider finite dimensional problems, since we intend to use probabilistic techniques for which the Euclidean space is the most standard setting. Accordingly, we assume the solution vector $X \in \mathbb{R}^{d}$, the linear model $A \in \mathbb{R}^{m \times d}$, and the datum $Y \in \mathbb{R}^{m}$. In the following we denote with $\|Z\|$ the Euclidean norm of a vector $Z \in \mathbb{R}^{N}$. One of the most widely used regularization approaches is realized by minimizing the following, so-called, Tikhonov functional

$$
\min _{z \in \mathbb{R}^{d}}\|A z-Y\|^{2}+\alpha\|z\|^{2}
$$

with $\alpha \in(0,+\infty)$. The regularized solution $Z^{\alpha}:=Z^{\alpha}(Y)$ of such minimization procedure is unique. In this context, the regularization scheme represents a trade-off between the accuracy of fitting the data $Y$ and the complexity of the solution, measured by a ball in $\mathbb{R}^{d}$ with radius depending on the regularization parameter $\alpha$. Therefore, the choice of the regularization parameter $\alpha$ is very crucial to identify the best possible regularized solution, which does not overfit the noise. This issue still remains one of the most delicate aspects of this approach and other regularization schemes. Clearly the best possible parameter minimizes the discrepancy between $Z^{\alpha}$ and the solution $X$

$$
\alpha^{*}=\arg \min _{\alpha \in(0,+\infty)}\left\|Z^{\alpha}-X\right\|
$$

Unfortunately, we usually have neither access to the solution $X$ nor to information about the noise, for instance, we might not be aware of the noise level $\|\eta\|$. Hence, for determining a possible good approximation to the optimal regularization parameter several approaches have been proposed, which can be categorized into three classes

- A priori parameter choice rules based on the noise level and some known "smoothness" of the solution encoded in terms, e.g., of the so-called source condition (Engl et al., 1996);

- A posteriori parameter choice rules based on the datum $Y$ and the noise level;

- A posteriori parameter choice rules based exclusively on the datum $Y$ or, the so-called, heuristic parameter choice rules. 
For the latter two categories there are by now a multitude of approaches. Below we recall the most used and relevant of them, indicating in square brackets their alternative names, accepted in different scientific communities. In most cases, the names we provide are the descriptive names originally given to the methods. However, in a few cases, there was no original name, and, to achieve consistency in the naming, we have chosen an appropriate one, reflecting the nature of the method. We mention, for instance, (transformed/modified) discrepancy principle [Raus-Gfrerer rule, minimum bound method]; monotone error rule; (fast/hardened) balancing principle also for white noise; quasi-optimality criterion; L-curve method; modified discrepancy partner rule [Hanke-Raus rule]; extrapolated error method; normalized cumulative periodogram method; residual method; generalized maximum likelihood; (robust/strong robust/modified) generalized cross-validation. Considering the large number of available parameter choice methods, there are relatively few comparative studies and we refer to (Bauer and Lukas, 2011) for a rather comprehensive discussion on their differences, pros and contra. One of the features which is common to most of the a posteriori parameter choice rules is the need of solving (3) multiple times for different values of the parameters $\alpha$, often selected out of a conveniently pre-defined grid.

In this paper, we intend to study a novel, data-driven, regularization method, which also yields approximations to the optimal parameter in Tikhonov regularization. After an offline learning phase, whose complexity scales at most algebraically with the dimensionality of the problem, our method does not require any additional knowledge of the noise level; the computation of a near-optimal regularization parameter can be performed very efficiently by solving the regularization problem (3) only a moderated amount times, see Section 6 for a discussion on the computational complexity. In particular cases, no solution of (3) is actually needed, see Section 5. Not being based on the noise level, our approach fits into the class of heuristic parameter choice rules (Kindermann, 2011). The approach aims at employing the framework of supervised machine learning to the problem of approximating the highdimensional function, which maps noisy data into the corresponding optimal regularization parameter. More precisely, we assume that we are allowed to see a certain number $n$ of examples of solutions $X_{i}$ and corresponding noisy data $Y_{i}=A X_{i}+\eta_{i}$, for $i=1, \ldots, n$. For all of these examples, we are clearly capable to compute the optimal regularization parameters as in the following scheme

$$
\begin{aligned}
\left(X_{1}, Y_{1}\right) \rightarrow & \alpha_{1}^{*}=\arg \min _{\alpha \in(0,+\infty)}\left\|Z^{\alpha}\left(Y_{1}\right)-X_{1}\right\| \\
\left(X_{2}, Y_{2}\right) \rightarrow & \alpha_{2}^{*}=\arg \min _{\alpha \in(0,+\infty)}\left\|Z^{\alpha}\left(Y_{2}\right)-X_{2}\right\| \\
\cdots & \cdots \\
\left(X_{n}, Y_{n}\right) \rightarrow & \alpha_{n}^{*}=\arg \min _{\alpha \in(0,+\infty)}\left\|Z^{\alpha}\left(Y_{n}\right)-X_{n}\right\| \\
(? ?, Y) & \rightarrow \bar{\alpha}
\end{aligned}
$$

Denote $\mu$ the joint distribution of the empirical samples $\left(Y_{1}, \alpha_{1}^{*}\right), \ldots,\left(Y_{n}, \alpha_{n}^{*}\right)$. Were its conditional distribution $\mu(\cdot \mid Y)$ with respect to the first variable $Y$ very much concentrated (for instance, when $\int_{0}^{\infty}(\alpha-\bar{\alpha})^{q} d \mu(\alpha \mid Y)$ is very small for $q \geq 1$ and for variable $Y$ ), then 
we could design a proper regression function

$$
\mathcal{R}: Y \mapsto \bar{\alpha}:=\mathcal{R}(Y)=\int_{0}^{\infty} \alpha d \mu(\alpha \mid Y) .
$$

Such a mapping would allow us, to a given new datum $Y$ (without given solution!), to associate the corresponding parameter $\bar{\alpha}$ not too far from the true optimal one $\alpha^{*}$, at least with high probability. We illustrate schematically this theoretical framework in Figure 1.

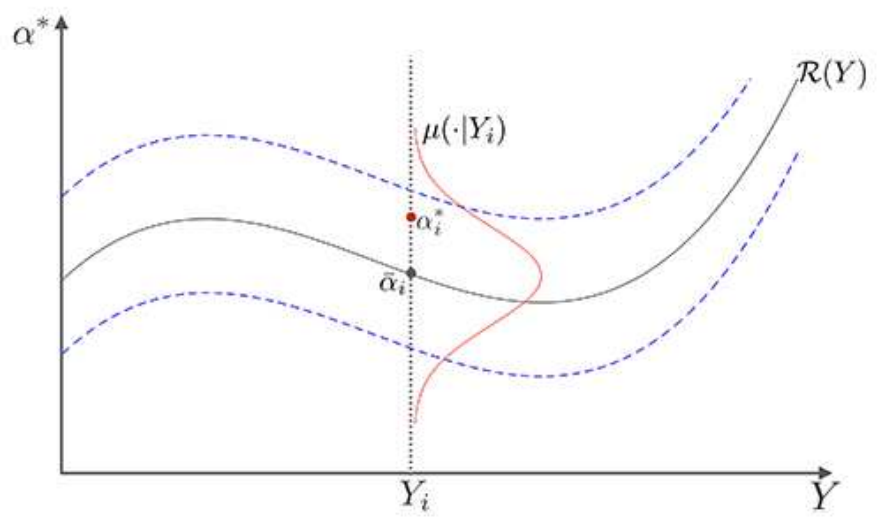

Figure 1: Learning optimal regularization parameters from previously observed samples by approximation of the regression function $\mathcal{R}$.

At a first glance, this setting may seem quite hopeless. First of all, one should establish the concentration of the conditional distribution generating $\alpha^{*}$ given $Y$. Secondly, even if we assume that the regression function $\mathcal{R}$ is very smooth, the vectors $Y$ belong to the space $\mathbb{R}^{m}$ and the number of observations $n$ required to learn such a function need to scale exponentially with the dimension $m$ (Novak and Woźniakowski, 2009). It is clear that we cannot address neither of the above issues in general. The only hope is that the solutions are statistically distributed in a concentrated manner over smooth sets of lower dimension $h \ll m$ and the noise has also a concentrated distribution, so that the corresponding data $Y$ are concentrated around lower-dimensional sets as well. And luckily these two assumptions are to a certain extent realistic.

By now, the assumption that the possible solutions belong to a lower-dimensional set of $\mathbb{R}^{d}$ has become an important prior for many signal and image processing tasks. For instance, were solutions natural images, then it is known that images can be represented as nearly-sparse coefficient vectors with respect to shearlets expansions (Kutyniok and Labate, 2012). Hence, in this case the set of possible solutions can be stylized as a union of lower-dimensional linear subspaces, consisting of sparse vectors (Mallat, 2009). In other situations, it is known that the solution set can be stylized, at least locally, as a smooth lower-dimensional nonlinear manifold $\mathcal{V}$ (Allard et al., 2012; Chen et al., 2013; Little et al., 2017). Also in this case, at least locally, it is possible to approximate the solution set by means of affine lower-dimensional sets, representing tangent spaces to the manifold. Hence, the a priori knowledge that the solution is belonging to some special (often nonlinear) set 
should also be taken into account when designing the regularization method.

In this paper, we want to show very rigorously how one can construct, from a relatively small number of previously observed examples, an approximation $\widehat{\mathcal{R}}$ to the regression function $\mathcal{R}$, which is mapping a noisy datum into a good approximation to the optimal Tikhonov regularization parameter. To this end, we assume the solutions to be distributed sub-gaussianly over a linear subspace $\mathcal{V} \subset \mathbb{R}^{d}$ of dimension $h \ll m$ and the noise $\eta$ to be also sub-gaussian. The first statistical assumption is perhaps mostly technical to allow us to provide rigorous estimates. Let us describe the method of computation as follows. We introduce the $m \times m$ noncentered covariance matrix built from the noisy measurements

$$
\widehat{\Sigma}_{n}=\frac{1}{n} \sum_{i=1}^{n} Y_{i} \otimes Y_{i},
$$

and we denote by $\widehat{\Pi}_{n}$ the projections onto the vector space spanned by the first most relevant eigenvectors of $\widehat{\Sigma}_{n}$. Furthermore, we set $\widehat{\alpha}_{n} \in(0,+\infty)$ as the minimizer of

$$
\min _{\alpha \in(0,+\infty)}\left\|Z^{\alpha}-A^{\dagger} \widehat{\Pi}_{n} Y\right\|^{2}
$$

where $A^{\dagger}$ is the pseudo-inverse. We define

$$
\widehat{\mathcal{R}}(Y)=\widehat{\alpha}_{n}
$$

and we claim that this is actually a good approximation, up to noise level, to $\mathcal{R}$ as soon as $n$ is large enough, without incurring in the curse of dimensionality (i.e., without exponential dependency of the computational complexity on $d$ ). More precisely, we prove that, for a given $\tau>0$, with probability greater than $1-6 \mathrm{e}^{-\tau^{2}}$, we have that

$$
\left\|Z^{\widehat{\alpha}_{n}}-X\right\| \leq\left\|Z^{\alpha^{*}}-X\right\|+\frac{1}{\sigma_{d}} B(n, \tau, \sigma),
$$

where $\sigma_{d}$ is the smallest positive singular value of $A$. Let us stress that $B(n, \tau, \sigma)$ gets actually small for small $\sigma$ and for $n=\mathcal{O}\left(m \times h\right.$ ) (see formula (33)) and $\widehat{\mathcal{R}}(Y)=\widehat{\alpha}_{n}$ is $\sigma$-optimal. We provide an explicit expression for $B$ in Proposition 6 . In the special case where $A=I$ we derive a bound on the difference between the learned parameter $\widehat{\alpha}_{n}$ and the optimal parameter $\alpha^{*}$, see Theorem 12 , justifying even more precisely the approximation $\widehat{\mathcal{R}}(Y)=\widehat{\alpha}_{n} \approx \alpha^{*}=\mathcal{R}(Y)$.

The paper is organized as follows: After introducing some notation and problem set-up in the next section, we provide the accuracy bounds on the learned estimators with respect to their distribution dependent counterparts in Section 3. For the special case $A=I$ we provide an explicit bound on the difference between the learned and the optimal regularization parameter and discuss the amount of samples needed for an accurate learning in Section 4. We also exemplify the presented theoretical results with a few numerical illustrations. Section 5 provides explicit formulas by means of numerical linearization for the parameter learning. Section 6 offers a snapshot of the main contributions and presents a list of open questions for future work. Finally, Appendix A and Appendix B contain some background information on perturbation theory for compact operators, the sub-gaussian random variables, and proofs of some technical theorems, which are valuable for understanding the scope of the paper. 


\section{Setting}

This section presents some background material and sets the notation for the rest of the work. First, we fix some notation. The Euclidean norm of a vector $v$ is denoted by $\|v\|$ and the Euclidean scalar product between two vectors $v, w$ by $\langle v, w\rangle$. We denote with $S^{d-1}$ the Euclidean unit sphere in $\mathbb{R}^{d}$. If $M$ is a matrix, $M^{T}$ denotes its transpose, $M^{\dagger}$ the pseudo-inverse, $M^{\dagger k}=\left(M^{\dagger}\right)^{k}$ and $\|M\|$ its spectral norm. Furthermore, $\operatorname{ker} M$ and $\operatorname{ran} M$ are the null space and the range of $M$ respectively. For a square-matrix $M$, we use $\operatorname{Tr}(M)$ to denote its trace. If $v$ and $w$ are vectors (possibly of different length), $v \otimes w$ is the rank one matrix with entries $(v \otimes w)_{i j}=v_{i} w_{j}$.

Given a random vector $\xi \in \mathbb{R}^{d}$, its noncentered covariance matrix is denoted by

$$
\Sigma_{\xi}=\mathbb{E}[\xi \otimes \xi]
$$

which is a positive matrix satisfying the following property

$$
\operatorname{ran} \Sigma_{\xi}=\left(\operatorname{ker} \Sigma_{\xi}\right)^{\perp}=\operatorname{span}\left\{x \in \mathbb{R}^{d} \mid \mathbb{P}[\xi \in B(x, r)]>0 \forall r>0\right\},
$$

here $B(x, r)$ denotes the ball of radius $r$ with the center at $x$. A random vector $\xi$ is called sub-gaussian if

$$
\|\xi\|_{\psi_{2}}:=\sup _{v \in S^{d-1}} \sup _{q \geq 1} q^{-\frac{1}{2}} \mathbb{E}\left[|\langle\xi, v\rangle|^{q}\right]^{\frac{1}{q}}<+\infty .
$$

The value $\|\xi\|_{\psi_{2}}$ is called the sub-gaussian norm of $\xi$ and the space of sub-gaussian vectors becomes a normed vector space (Vershynin, 2012). Appendix B reviews some basic properties about sub-gaussian vectors.

We consider the following class of inverse problems.

Assumption 1 In the statistical linear inverse problem

$$
Y=A X+\sigma W
$$

the following conditions hold true:

a) $A$ is an $m \times d$-matrix with norm $\|A\|=1$;

b) the signal $X \in \mathbb{R}^{d}$ is a sub-gaussian random vector with $\|X\|_{\psi_{2}}=1$;

c) the noise $W \in \mathbb{R}^{m}$ is a sub-gaussian centered random vector independent of $X$ with $\|W\|_{\psi_{2}}=1 / \sqrt{2}$ and with the noise level $0<\sigma<\sqrt{2}$;

d) the covariance matrix $\Sigma_{X}$ of $X$ has a low rank matrix, i.e.,

$$
\operatorname{rank}\left(\Sigma_{X}\right)=h \ll d
$$

We add some comments on the above conditions. The normalisation assumptions on $\|A\|$, $\|X\|_{\psi_{2}}$ and $\|W\|_{\psi_{2}}$ are stated only to simplify the bounds. They can always be satisfied by rescaling $A, X$ and $W$ and our results hold true by replacing $\sigma$ with $\sqrt{2}\|W\|_{\psi_{2}}\|A\|^{-1}\|X\|_{\psi_{2}}^{-1} \sigma$. The upper bound on $\sigma$ reflects the intuition that $\sigma W$ is a small perturbation of the noiseless problem. 
Condition d) means that $X$ spans a low dimensional subspace of $\mathbb{R}^{d}$. Indeed, by (4) condition $\mathrm{d}$ ) is equivalent to the fact that the vector space

$$
\mathcal{V}=\operatorname{ran} \Sigma_{X}=\operatorname{span}\left\{x \in \mathbb{R}^{d} \mid \mathbb{P}[X \in B(x, r)]>0 \text { for all } r>0\right\}
$$

is an $h$-dimensional subspace and $h$ is the dimension of the minimal subspace containing $X$ with probability 1 , i.e.,

$$
h=\min _{\mathcal{K}} \operatorname{dim} \mathcal{K},
$$

where the minimum is taken over all subspaces $\mathcal{K} \subset \mathbb{R}^{d}$ such that $\mathbb{P}[X \in \mathcal{K}]=1$.

We write $a \lesssim b$ if there exists an absolute constant $C$ such that $a \leq C b$. By absolute we mean that it holds for all the problems $Y=A X+\sigma W$ satisfying Assumption 1, in particular, it is independent of $d, m$ and $h$.

The datum $Y$ depends only on the projection $X^{\dagger}$ of $X$ onto $\operatorname{ker} A^{\perp}$ and $Z^{\alpha}$ as solutions of (3) also belong to ker $A^{\perp}$. Therefore, we can always assume, without loss of generality, for the rest of the paper that $A$ is injective by replacing $X$ with $X^{\dagger}$, which is a sub-gaussian random vector, and $\mathbb{R}^{d}$ with $\operatorname{ker} A^{\perp}$.

Since $A$ is injective, $\operatorname{rank}(A)=d$ and we define the singular value decomposition of $A$ by $\left(u_{i}, v_{i}, \sigma_{i}\right)_{i=1}^{d}$, so that $A=U D V^{T}$ or

$$
A v_{i}=\sigma_{i} u_{i}, \quad i=1, \ldots, d,
$$

where $\sigma_{1} \geq \sigma_{2} \geq \cdots \geq \sigma_{d}>0$. Since $\|A\|=1$, clearly $\sigma_{1}=1$. Furthermore, let $Q$ be the projection onto the $\operatorname{span}\left\{u_{1}, \ldots, u_{d}\right\}$, so that $Q A=A$, and we have the decomposition

$$
Q=A A^{\dagger}
$$

Recalling (6), since $A$ is now assumed injective and

$$
\Sigma_{A X}=\mathbb{E}[A X \otimes A X]=A \Sigma_{X} A^{T}
$$

then

$$
\mathcal{W}=\operatorname{ran} \Sigma_{A X}=\left(\operatorname{ker} \Sigma_{A X}\right)^{\perp}=A \mathcal{V}
$$

and, by condition d) in Assumption 1, we have as well $\operatorname{dim} \mathcal{W}=h$.

We denote by $\Pi$ the projection onto $\mathcal{W}$ and by

$$
p=\max \left\{i \in\{1, \ldots, d\} \mid \Pi u_{i} \neq 0\right\},
$$

so that, with probability 1 ,

$$
\Pi A X=A X \quad \text { and } \quad X=\sum_{i=1}^{p}\left\langle X, v_{i}\right\rangle v_{i} .
$$

Finally, the random vectors $\eta=\sigma W, A X$, and $Y$ are sub-gaussian and take value in $\mathbb{R}^{m}$, $\mathcal{W}$, and $\mathbb{R}^{m}$, respectively, with

$$
\|A X\|_{\psi_{2}} \leq\left\|A^{T}\right\|\|X\|_{\psi_{2}}=1 \quad\|Y\|_{\psi_{2}} \leq\|A X\|_{\psi_{2}}+\sigma\|W\|_{\psi_{2}} \leq 2
$$


since, by Assumption $1,\|A\|=1$ and $\sigma \leq \sqrt{2}$.

For any $t \in[0,1]$ we set $Z^{t}$ as the solution of the minimization problem

$$
\min _{z \in \mathbb{R}^{d}}\left(t\|A z-Y\|^{2}+(1-t)\|z\|^{2}\right),
$$

which is the solution of the Tikhonov functional

$$
\min _{z \in \mathbb{R}^{d}}\|A z-Y\|^{2}+\alpha\|z\|^{2}
$$

with $\alpha=(1-t) / t \in[0,+\infty]$.

For $t<1$, the solution is unique, for $t=1$ the minimizer is not unique and we set

$$
Z^{1}=A^{\dagger} Y \text {. }
$$

The explicit form of the solution of (12) is given by

$$
\begin{aligned}
Z^{t} & =t\left(t A^{T} A+(1-t) I\right)^{-1} A^{T} Y \\
& =\sum_{i=1}^{d} \frac{t \sigma_{i}}{t \sigma_{i}^{2}+(1-t)}\left\langle Y, u_{i}\right\rangle v_{i} \\
& =\sum_{i=1}^{d}\left(\frac{t \sigma_{i}^{2}}{t \sigma_{i}^{2}+(1-t)}\left\langle X, v_{i}\right\rangle+\frac{t \sigma_{i}}{t \sigma_{i}^{2}+(1-t)}\left\langle\eta, u_{i}\right\rangle\right) v_{i},
\end{aligned}
$$

which shows that $Z^{t}$ is also a sub-gaussian random vector.

We seek for the optimal parameter $t^{*} \in[0,1]$ that minimizes the reconstruction error

$$
\min _{t \in[0,1]}\left\|Z^{t}-X\right\|^{2}
$$

Since $X$ is not known, the optimal parameter $t^{*}$ can not be computed. We assume that we have at disposal a training set of $n$-independent noisy data

$$
Y_{1}, \ldots, Y_{n}
$$

where $Y_{i}=A X_{i}+\sigma W_{i}$, and each pair $\left(X_{i}, W_{i}\right)$ is distributed as $(X, W)$, for $i=1, \ldots, n$.

We introduce the $m \times m$ empirical covariance matrix

$$
\widehat{\Sigma}_{n}=\frac{1}{n} \sum_{i=1}^{n} Y_{i} \otimes Y_{i},
$$

and we denote by $\widehat{\Pi}_{n}$ the projections onto the vector space spanned by the first $h$-eigenvectors of $\widehat{\Sigma}_{n}$, where the corresponding (repeated) eigenvalues are ordered in a nonincreasing way.

Remark 1 The well-posedness of the empirical realization $\widehat{\Pi}_{n}$ in terms of spectral gap at the h-th eigenvalue will be given in Theorem 3, where we show that for $n$ large enough the $h+1$-th eigenvalue is strictly smaller than the $h$-th eigenvalue. 
We define the empirical estimators of $X$ and $\eta$ as

$$
\widehat{X}=A^{\dagger} \widehat{\Pi}_{n} Y \quad \text { and } \quad \widehat{\eta}=\left(Y-\widehat{\Pi}_{n} Y\right),
$$

so that, by Equation (7),

$$
A \widehat{X}+Q \widehat{\eta}=Q Y
$$

Furthermore, we set $\widehat{t}_{n} \in[0,1]$ as the minimizer of

$$
\min _{t \in[0,1]}\left\|Z^{t}-\widehat{X}\right\|^{2}
$$

If $\widehat{X}$ is close to $X$, we expect that the solution $Z^{\widehat{t}_{n}}$ has a reconstruction error close to the minimum value. In the following sections, we study the statistical properties of $\widehat{t}_{n}$. However, we first provide some a priori information on the optimal regularization parameter $t^{*}$.

\subsection{Distribution dependent quantities}

We define the function $t \mapsto\|R(t)\|^{2}$, where

$$
R(t)=Z^{t}-X \quad t \in[0,1]
$$

is the reconstruction error vector. Clearly, the function $t \mapsto\|R(t)\|^{2}$ is continuous, so that a global minimizer $t^{*}$ always exists in the compact interval $[0,1]$.

Define for all $t \in[0,1]$ the $d \times d$ matrix

$$
B(t)=t A^{T} A+(1-t) I=\sum_{i=1}^{d}\left(t \sigma_{i}^{2}+1-t\right) v_{i} \otimes v_{i}
$$

which is invertible since $A$ is injective and its inverse is

$$
B(t)^{-1}=\sum_{i=1}^{d} \frac{1}{t \sigma_{i}^{2}+1-t} v_{i} \otimes v_{i}
$$

Furthermore, $B(t)$ and $B(t)^{-1}$ are smooth functions of the parameter $t$ and

$$
B^{\prime}(t)=\left(A^{T} A-I\right) \quad\left(B(t)^{-1}\right)^{\prime}=-B(t)^{-2}\left(A^{T} A-I\right) .
$$

Since $Y=A X+\eta$, expression (13) gives

$$
\begin{aligned}
R(t) & =t B(t)^{-1} A^{T} Y-X \\
& =t B(t)^{-1} A^{T}(A X+\eta)-X \\
& =B(t)^{-1}\left(t A^{T} A X-B(t) X+t A^{T} \eta\right) \\
& =B(t)^{-1}\left(-(1-t) X+t A^{T} \eta\right) .
\end{aligned}
$$

Hence,

$$
\begin{aligned}
\|R(t)\|^{2} & =\left\|B(t)^{-1}\left(-(1-t) X+t A^{T} \eta\right)\right\|^{2} \\
& =\sum_{i=1}^{d}\left(\frac{-(1-t) \xi_{i}+t \sigma_{i} \nu_{i}}{t \sigma_{i}^{2}+(1-t)}\right)^{2}
\end{aligned}
$$


where for all $i=1, \ldots, d$

$$
\xi_{i}=\left\langle X, v_{i}\right\rangle \quad \nu_{i}=\left\langle\eta, u_{i}\right\rangle .
$$

In order to characterize $t^{*}$ we may want to seek it among the zeros of the following function

$$
H(t)=\frac{1}{2} \frac{d}{d t}\left\|Z^{t}-X\right\|^{2}=\left\langle R(t), R^{\prime}(t)\right\rangle .
$$

Taking into account (18), the differentiation of (19) is given by

$$
\begin{aligned}
R^{\prime}(t) & =B(t)^{-1} A^{T} Y-t B(t)^{-2}\left(A^{T} A-I\right) A^{T} Y \\
& =B(t)^{-2}\left(B(t)-t\left(A^{T} A-I\right)\right) A^{T} Y \\
& =B(t)^{-2} A^{T} Y
\end{aligned}
$$

so that

$$
\begin{aligned}
H(t) & =\left\langle A B(t)^{-3}\left(-(1-t) X+t A^{T} \eta\right), A X+\eta\right\rangle \\
& =\sum_{i=1}^{d} \sigma_{i} \frac{-(1-t) \xi_{i}+t \sigma_{i} \nu_{i}}{\left(t \sigma_{i}^{2}+(1-t)\right)^{3}}\left(\xi_{i} \sigma_{i}+\nu_{i}\right) \\
& =\sum_{i=1}^{d} \sigma_{i} \xi_{i}\left(\xi_{i} \sigma_{i}+\nu_{i}\right) \frac{\left(\sigma_{i} \nu_{i} \xi_{i}^{-1}+1\right) t-1}{\left(1-\left(1-\sigma_{i}^{2}\right) t\right)^{3}} \\
& =\sum_{i=1}^{d} \sigma_{i} \alpha_{i} h_{i}(t),
\end{aligned}
$$

where $\alpha_{i}=\xi_{i}\left(\sigma_{i} \xi_{i}+\nu_{i}\right)$ and $h_{i}(t)=\frac{\left(\sigma_{i} \nu_{i} \xi_{i}^{-1}+1\right) t-1}{\left(1-\left(1-\sigma_{i}^{2}\right) t\right)^{3}}$.

We observe that

a) if $t=0$ (i.e., $\alpha=+\infty), B(0)=I$, then

$$
H(0)=-\|A X\|^{2}-\langle A X, \eta\rangle,
$$

which is negative if $\|\Pi \eta\| \leq\|A X\|$, i.e., for

$$
\sigma \leq \frac{\|A X\|}{\|\Pi W\|} .
$$

Furthermore, by construction,

$$
\mathbb{E}[H(0)]=-\operatorname{Tr}\left(\Sigma_{A X}\right)<0
$$

b) if $t=1$ (i.e., $\alpha=0), B(1)=A^{T} A$ and

$$
\begin{aligned}
H(1) & =\left\langle A\left(A^{T} A\right)^{-3} A^{T} \eta, A X+\eta\right\rangle \\
& =\left\|\left(A A^{T}\right)^{\dagger} \eta\right\|^{2}+\left\langle\left(A A^{T}\right)^{\dagger} \eta,\left(A^{T}\right)^{\dagger} X\right\rangle,
\end{aligned}
$$


which is positive if $\left\|\left(A A^{T}\right)^{\dagger} \eta\right\| \geq\left\|\left(A^{T}\right)^{\dagger} X\right\|$, for example, when

$$
\sigma \geq \sigma_{d} \frac{\|X\|}{\left|\left\langle W, u_{d}\right\rangle\right|}
$$

Furthermore, by construction,

$$
\mathbb{E}[H(1)]=\operatorname{Tr}\left(\Sigma_{\left(A A^{T}\right)^{\dagger} \eta}\right)>0 .
$$

Hence, if the noise level satisfies

$$
\frac{\|X\|}{\left|\left\langle W, u_{d}\right\rangle\right|} \leq \sigma \leq \frac{\|A X\|}{\|\Pi W\|}
$$

the minimizer $t^{*}$ is in the open interval $(0,1)$ and it is a zero of $H(t)$. If $\sigma$ is too small, there is no need of regularization since we are dealing with a finite dimensional problem. On the opposite side, if $\sigma$ is too big, the best solution is the trivial one, i.e., $Z^{t^{*}}=0$.

\subsection{Empirical quantities}

We replace $X$ and $\eta$ with their empirical counterparts defined in (16). By Equation (17) and reasoning as in Equation (19), we obtain

$$
\begin{aligned}
\widehat{R}_{n}(t) & =Z^{t}-\widehat{X} \\
& =t B(t)^{-1} A^{T} Q Y-\widehat{X} \\
& =B(t)^{-1}\left(-(1-t) \widehat{X}+t A^{T} \widehat{\eta}\right),
\end{aligned}
$$

and

$$
\begin{aligned}
\left\|\widehat{R}_{n}(t)\right\|^{2} & =\left\|B(t)^{-1}\left(-(1-t) \widehat{X}+t A^{T} \widehat{\eta}\right)\right\|^{2} \\
& =\sum_{i=1}^{d}\left(\frac{-(1-t) \widehat{\xi}_{i}+t \sigma_{i} \widehat{\nu}_{i}}{t \sigma_{i}^{2}+(1-t)}\right)^{2},
\end{aligned}
$$

where for all $i=1, \ldots, d$

$$
\widehat{\xi}_{i}=\left\langle\widehat{X}, v_{i}\right\rangle \quad \text { and } \quad \widehat{\nu}_{i}=\left\langle\widehat{\eta}, u_{i}\right\rangle .
$$

Clearly,

$$
\widehat{R}_{n}^{\prime}(t)=R^{\prime}(t)=B(t)^{-2} A^{T} Q Y
$$

From (21), we get

$$
\begin{aligned}
\widehat{H}_{n}(t) & =\left\langle\widehat{R}_{n}(t), \widehat{R}_{n}^{\prime}(t)\right\rangle \\
& \left.=\left\langle B(t)^{-3}\left(-(1-t) \widehat{X}+t A^{T} \widehat{\eta}\right), A^{T} A \widehat{X}+A^{T} \widehat{\eta}\right)\right\rangle \\
& =\sum_{i=1}^{d} \frac{-(1-t) \widehat{\xi}_{i}+t \sigma_{i} \widehat{\nu}_{i}}{\left(1-\left(1-\sigma_{i}^{2}\right) t\right)^{3}}\left(\widehat{\xi}_{i} \sigma_{i}^{2}+\sigma_{i} \widehat{\nu}_{i}\right), \\
& =\sum_{i=1}^{d} \sigma_{i} \widehat{\alpha}_{i} \widehat{h}_{i}(t),
\end{aligned}
$$


where $\widehat{\alpha}_{i}=\widehat{\xi}_{i}\left(\sigma_{i} \widehat{\xi}_{i}+\widehat{\nu}_{i}\right)$ and $\widehat{h}_{i}(t)=\frac{\left(\sigma_{i} \widehat{\nu}_{i} \widehat{\xi}_{i}^{-1}+1\right) t-1}{\left(1-\left(1-\sigma_{i}^{2}\right) t\right)^{3}}$.

An alternative form in terms of $Y$ and $\widehat{\Pi}_{n}$, which can be useful as a different numerical implementation, is

$$
\begin{aligned}
\widehat{H}_{n}(t) & =\left\langle B(t)^{-1}\left(t A^{T}\left(Y-\widehat{\Pi}_{n} Y\right)-(1-t) A^{\dagger} \widehat{\Pi}_{n} Y\right), B(t)^{-2} A^{T} Y\right\rangle \\
& =\left\langle t A A^{T}\left(Y-\widehat{\Pi}_{n} Y\right)-(1-t) Q \widehat{\Pi}_{n} Y,\left(t A A^{T}+(1-t) I\right)^{\dagger 3} Q Y\right\rangle \\
& =\left\langle t A A^{T}\left(Y-\widehat{\Pi}_{n} Y\right)-(1-t) \widehat{\Pi}_{n} Y,\left(t A A^{T}+(1-t) I\right)^{\dagger 3} Q Y\right\rangle .
\end{aligned}
$$

As for $t^{*}$, the minimizer $\widehat{t}_{n}$ of the function $t \mapsto\left\|\widehat{R}_{n}(t)\right\|^{2}$ always exists in [0,1] and, for $\sigma$ in the range of interest, it is in the open interval $(0,1)$, so that it is a zero of the function $\widehat{H}_{n}(t)$.

\section{Concentration inequalities}

In this section, we bound the difference between the empirical estimators and their distribution dependent counterparts.

By (8) and item d) of Assumption 1, the covariance matrix $\Sigma_{A X}$ has rank $h$ and, we set $\lambda_{\min }$ to be the smallest non-zero eigenvalue of $\Sigma_{A X}$. Furthermore, we denote by $\Pi^{Y}$ the projection from $\mathbb{R}^{m}$ onto the vector space spanned by the eigenvectors of $\Sigma_{Y}$ with corresponding eigenvalue greater than $\lambda_{\min } / 2$.

The following proposition shows that $\Pi^{Y}$ is close to $\Pi$ if the noise level is small enough.

Proposition 2 If $\sigma^{2}<\lambda_{\min } / 4$, then $\operatorname{dim} \operatorname{ran} \Pi^{Y}=h$ and

$$
\left\|\Pi^{Y}-\Pi\right\| \leq \frac{2 \sigma^{2}}{\lambda_{\min }}
$$

Proof Since $A X$ and $W$ are independent and $W$ has zero mean, then

$$
\Sigma_{Y}=\Sigma_{A X}+\sigma^{2} \Sigma_{W}
$$

Since $\Sigma_{W}$ is a positive matrix and $W$ is a sub-gaussian vector satisfying (11), with the choice $q=2$ in (5), we have

$$
\left\|\Sigma_{W}\right\|=\sup _{v \in S^{m-1}}\left\langle\Sigma_{W} v, v\right\rangle=\sup _{v \in S^{m-1}} \mathbb{E}\left[\langle W, v\rangle^{2}\right] \leq 2\|W\|_{\psi_{2}}^{2}=1
$$

so that $\left\|\Sigma_{Y}-\Sigma_{A X}\right\| \leq \sigma^{2}<\lambda_{\min } / 4$.

We now apply Proposition 15 with $\mathcal{A}=\Sigma_{A X}$ and eigenvalues $\left(\alpha_{j}\right)_{j}$ and projections ${ }^{1}$ $\left(P_{j}\right)_{j}$, and $\mathcal{B}=\Sigma_{Y}$ with eigenvalues $\left(\beta_{\ell}\right)_{\ell}$ and projections $\left(Q_{\ell}\right)_{\ell}$. We choose $j$ such that $\alpha_{j}=\lambda_{\min }$ so that $\alpha_{j+1}=0, P_{j}=\Pi$ and, by (8),

$$
\operatorname{dim} \operatorname{ran} P_{j}=\operatorname{dim} \operatorname{ran} \Pi=\operatorname{dim} \operatorname{ran} \Sigma_{A X}=h .
$$

1. In the statement of Proposition 15 the eigenvalues are counted without their multiplicity and ordered in a decreasing way and each $P_{j}$ is the projection onto the vector space spanned by the eigenvectors with corresponding eigenvalue greater or equal than $\alpha_{j}$. 
Then there exists $\ell$ such that $\beta_{\ell+1}<\lambda_{\min } / 2<\beta_{\ell}$, so that $Q_{\ell}=\Pi^{Y}$ and it holds that $\operatorname{dim} \operatorname{ran} \Pi^{Y}=\operatorname{dim} \operatorname{ran} P_{j}=h$. Finally, (45) implies (24) since $\alpha_{h+1}=0$.

Recall that $\widehat{\Pi}_{n}$ is the projection onto the vector space spanned by the first $h$-eigenvectors of $\widehat{\Sigma}_{n}$ defined by (15).

Theorem 3 Given $\tau>0$ with probability greater than $1-2 \mathrm{e}^{-\tau^{2}}, \widehat{\Pi}_{n}$ coincides with the projection onto the vector space spanned by the eigenvectors of $\widehat{\Sigma}_{n}$ with corresponding eigenvalue greater than $\lambda_{\min } / 2$. Furthermore

$$
\left\|\widehat{\Pi}_{n}-\Pi\right\| \lesssim \frac{1}{\lambda_{\min }}\left(\sqrt{\frac{m}{n}}+\frac{\tau}{\sqrt{n}}+\sigma^{2}\right),
$$

provided that

$$
\begin{aligned}
n & \gtrsim(\sqrt{m}+\tau)^{2} \max \left\{\frac{64}{\lambda_{\min }^{2}}, 1\right\} \\
\sigma^{2} & <\frac{\lambda_{\min }}{8} .
\end{aligned}
$$

Proof We apply Theorem 20 with $\xi_{i}=Y_{i}$ and

$$
\delta=C \sqrt{\frac{m}{n}}+\frac{\tau}{\sqrt{n}} \leq \min \left\{1, \lambda_{\min } / 8\right\} \leq 1,
$$

by $(25)$. Since $\delta^{2} \leq \delta$, with probability greater than $1-2 \mathrm{e}^{-\tau^{2}}$,

$$
\begin{aligned}
\left\|\widehat{\Sigma}_{n}-\Sigma_{A X}\right\| & \leq\left\|\widehat{\Sigma}_{n}-\Sigma_{Y}\right\|+\left\|\Sigma_{Y}-\Sigma_{A X}\right\| \\
& \leq C\left(\sqrt{\frac{m}{n}}+\frac{\tau}{\sqrt{n}}\right)+\sigma^{2} \\
& \leq \frac{\lambda_{\text {min }}}{8}+\frac{\lambda_{\text {min }}}{8}=\frac{\lambda_{\text {min }}}{4},
\end{aligned}
$$

where the last inequality follows by (28).

As in the proof of Proposition 2, we apply Proposition 15 with $\mathcal{A}=\Sigma_{A X}, \mathcal{B}=\widehat{\Sigma}_{n}$ and $\alpha_{j}=\lambda_{\min }$ to be the smallest eigenvalue of $\Sigma_{A X}$, so that $P_{j}=\Pi$ and $\operatorname{dim} \operatorname{ran} P_{j}=h$. Then, there exists a unique eigenvalue $\beta_{\ell}$ of $\widehat{\Sigma}_{n}$ such that $\beta_{\ell+1}<\lambda_{\min } / 2<\beta_{\ell}$ and $\operatorname{dim} \operatorname{ran} Q_{\ell}=\operatorname{dim} \operatorname{ran} P_{j}=h$. Then $Q_{\ell}=\widehat{\Pi}_{n}$ and (45) implies (26). Note that the constant $C$ depends on $\|Y\|_{\psi_{2}} \leq 2$ by (11), so that it becomes an absolute constant, when considering the worst case $\|Y\|_{\psi_{2}}=2$.

If $n$ and $\sigma$ satisfy (27), the above proposition shows that the empirical covariance matrix $\widehat{\Sigma}_{n}$ has a spectral gap around the value $\lambda_{\min } / 2$ and the number of eigenvector with corresponding eigenvalue greater than $\lambda_{\min } / 2$ is precisely $h$, so that $\widehat{\Pi}_{n}$ is uniquely defined. Furthermore, the dimension $h$ can be estimated by observing spectral gaps in the singular value decomposition of $\widehat{\Sigma}_{n}$.

If the number $n$ of samples goes to infinity, bound (26) does not converge to zero due to term proportional to the noise level $\sigma$. However, if the random noise $W$ is isotropic, we can improve the estimate. 
Theorem 4 Assume that $\Sigma_{W}=$ Id. Given $\tau>0$ with probability greater than $1-2 \mathrm{e}^{-\tau^{2}}$,

$$
\left\|\widehat{\Pi}_{n}-\Pi\right\| \lesssim \frac{1}{\lambda_{\min }}\left(\sqrt{\frac{m}{n}}+\frac{\tau}{\sqrt{n}}\right),
$$

provided that

$$
\begin{aligned}
n & \gtrsim(\sqrt{m}+\tau)^{2}\left\{1, \frac{16}{\lambda_{\min }^{2}}\right\} \\
\sigma^{2} & <\frac{\lambda_{\min }}{2} .
\end{aligned}
$$

Proof As in the proof of Proposition 2, we have that

$$
\Sigma_{Y}=\Sigma_{A X}+\sigma^{2} \Sigma_{W}=\Sigma_{A X}+\sigma^{2} \mathrm{Id}
$$

where the last equality follows from the assumption that the noise is isotropic. Hence, the matrices $\Sigma_{Y}$ and $\Sigma_{A X}$ have the same eigenvectors, whereas the corresponding eigenvalues are shifted by $\sigma^{2}$. Taking into account that $\lambda_{\min }$ is the smallest non-zero eigenvalue of $\Sigma_{A X}$ and denoted by $\left(\alpha_{j}\right)_{j=1}^{N}$ the eigenvalues of $\Sigma_{Y}$, it follows that there exists $j=1, \ldots, N$ such that

$$
\alpha_{1}>\alpha_{2}>\alpha_{j}=\lambda_{\min }+\sigma^{2} \quad \alpha_{j+1}=\ldots=\alpha_{N}=\sigma^{2}
$$

Furthermore, denoted by $P_{j}$ the projection onto the vector space spanned by the eigenvectors with corresponding eigenvalue greater or equal than $\alpha_{j}$, it holds that $\Pi=P_{j}$. By assumption $\sigma^{2}<\lambda_{\min } / 2$, so that $\Pi^{Y}=P_{j}=\Pi$ and, hence, $\operatorname{dim} \operatorname{ran} P_{j}=h$.

As in the proof of Theorem 3 , with probability $1-2 \mathrm{e}^{-\tau^{2}}$,

$$
\left\|\widehat{\Sigma}_{n}-\Sigma_{Y}\right\| \leq C\left(\sqrt{\frac{m}{n}}+\frac{\tau}{\sqrt{n}}\right)<\min \left\{1, \frac{\lambda_{\min }}{4}\right\}<\frac{\alpha_{h}-\alpha_{h+1}}{4},
$$

where $n$ is large enough, see (30). Then, there exists a unique eigenvalue $\beta_{\ell}$ of $\widehat{\Sigma}_{n}$ such that $\beta_{\ell+1}<\frac{\lambda_{\min }}{2}+\sigma^{2}<\beta_{\ell}$ and $\operatorname{dim} \operatorname{ran} Q_{\ell}=\operatorname{dim} \operatorname{ran} P_{j}=h$. Then $Q_{\ell}=\widehat{\Pi}_{n}$ and $(45)$ implies (29).

We need the following technical lemma.

Lemma 5 Given $\tau>0$, with probability greater than $1-4 \mathrm{e}^{-\tau^{2}}$, simultaneously it holds

$$
\|X\| \lesssim(\sqrt{h}+\tau) \quad\|Y\| \lesssim(\sqrt{h}+\sigma \sqrt{m}+\tau) \quad\|\Pi W\| \lesssim(\sqrt{h}+\tau) .
$$

Proof Since $X$ is a sub-gaussian random vector taking values in $\mathcal{V}$ with $h=\operatorname{dim} \mathcal{V}$, taking into account that $\|X\|_{\psi_{2}}=1$, bound (50) gives

$$
\|X\| \leq 9(\sqrt{h}+\tau)
$$

with probability greater than $1-2 \mathrm{e}^{-\tau^{2}}$. Since $W$ is a centered sub-gaussian random vector taking values in $\mathbb{R}^{m}$ and $\|W\|_{\psi_{2}} \leq 1$, by (51)

$$
\|W\| \leq 16(\sqrt{m}+\tau),
$$


with probability greater than $1-\mathrm{e}^{-\tau^{2}}$. Since $\|A\|=1$ and

$$
\|Y\| \leq\|A X\|+\sigma\|W\| \leq\|X\|+\sigma\|W\|,
$$

the first two bounds in (31) hold true with probability greater than $1-3 \mathrm{e}^{-\tau^{2}}$. Since $\Pi W$ is a centered sub-gaussian random vector taking values in $\mathcal{W}$ with $h=\operatorname{dim} \mathcal{W}$, and $\|\Pi W\|_{\psi_{2}} \leq 1$, by $(51)$

$$
\|\Pi W\| \leq 16(\sqrt{h}+\tau)
$$

with probability greater than $1-\mathrm{e}^{-\tau^{2}}$.

As a consequence, we have the following bound.

Proposition 6 Given $\tau>0$, if $n$ and $\sigma$ satisfy (27), then with probability greater than $1-6 \mathrm{e}^{-\tau^{2}}$

$$
\left\|\left(\Pi-\widehat{\Pi}_{n}\right) Y-\Pi \eta\right\| \lesssim B(n, \tau, \sigma),
$$

where

$$
\begin{aligned}
B(n, \tau, \sigma)= & \frac{1}{\lambda_{\min }} \sqrt{\frac{h m}{n}}+\sigma\left(\sqrt{h}+\frac{1}{\lambda_{\min }} \frac{m}{\sqrt{n}}\right)+\frac{\sigma^{2}}{\lambda_{\min }} \sqrt{h}+\frac{\sigma^{3}}{\lambda_{\min }} \sqrt{m}+ \\
& +\tau\left(\frac{1}{\lambda_{\min }} \sqrt{\frac{m}{n}}+\sigma\left(1+\frac{1}{\lambda_{\min }} \sqrt{\frac{m}{n}}\right)+\frac{\sigma^{2}}{\lambda_{\min }}\right)+\tau^{2} \frac{1}{\lambda_{\min }} \frac{1}{\sqrt{n}} .
\end{aligned}
$$

Proof Clearly,

$$
\left\|\left(\Pi-\widehat{\Pi}_{n}\right) Y-\Pi \eta\right\| \leq\left\|\Pi-\widehat{\Pi}_{n}\right\|\|Y\|+\sigma\|\Pi W\| .
$$

If (27) holds true, bounds (26) and (31) imply

$$
\left\|\left(\Pi-\widehat{\Pi}_{n}\right) Y-\Pi \eta\right\| \lesssim \frac{1}{\lambda_{\min }}\left(\sqrt{\frac{m}{n}}+\frac{\tau}{\sqrt{n}}+\sigma^{2}\right)(\sqrt{h}+\sigma \sqrt{m}+\tau)+\sigma(\sqrt{h}+\tau),
$$

with probability greater than $1-6 \mathrm{e}^{-\tau^{2}}$. By developing the brackets and taking into account that $\sqrt{h+m} \leq \sqrt{2 m},(32)$ holds true.

Remark 7 Usually in machine learning bounds of the type (32) are considered in terms of their expectation, e.g., with respect to $(X, Y)$. In our framework, this would amount to the following bound

$$
\begin{aligned}
& \mathbb{E}\left[\left\|\left(\Pi-\widehat{\Pi}_{n}\right) Y-\Pi \eta\right\| \mid Y_{1}, \ldots, Y_{n}\right] \lesssim \\
& \lesssim \frac{1}{\lambda_{\min }}\left(\sqrt{\frac{m}{n}}+\frac{\tau}{\sqrt{n}}+\sigma^{2}\right)(\sqrt{h}+\sigma \sqrt{m})+\sigma \sqrt{h},
\end{aligned}
$$

obtained by observing that $\mathbb{E}[\|Y\|] \leq \mathbb{E}[\|A\|\|X\|]+\sigma \mathbb{E}[\|W\|]$,

$$
\mathbb{E}[\|X\|]^{2} \leq \mathbb{E}\left[\|X\|^{2}\right]=\operatorname{Tr}\left(\Sigma_{X}\right) \leq 2 h\|X\|_{\psi_{2}}^{2} \lesssim h,
$$

and, by a similar computation,

$$
\mathbb{E}[\|W\|] \lesssim \sqrt{m} \quad \mathbb{E}[\|\Pi W\|] \lesssim \sqrt{h}
$$

Our bound (32) is much stronger and it holds in probability with respect to both the training set $Y_{1}, \ldots Y_{n}$ and the new pair $(X, Y)$. 
Our first result is a direct consequence of the estimate (32).

Theorem 8 Given $\tau>0$, with probability greater than $1-6 \mathrm{e}^{-\tau^{2}}$,

$$
\begin{aligned}
\|\widehat{X}-X\| & \lesssim \frac{1}{\sigma_{d}} B(n, \tau, \sigma) \\
\|Q \widehat{\eta}-Q \eta\| & \lesssim B(n, \tau, \sigma) \\
\left\|Z^{t_{n}}-X\right\|-\left\|Z^{t^{*}}-X\right\| & \lesssim \frac{1}{\sigma_{d}} B(n, \tau, \sigma) \\
\sup _{0 \leq t \leq 1}\left\|\widehat{R}_{n}(t)\right\|-\|R(t)\| \mid & \lesssim \frac{1}{\sigma_{d}} B(n, \tau, \sigma)
\end{aligned}
$$

provided that $n$ and $\sigma$ satisfy (27).

Proof By the first identity of (10)

$$
\begin{aligned}
X-\widehat{X} & =A^{\dagger} \Pi A X-A^{\dagger} \widehat{\Pi}_{n}(A X+\eta) \\
& =A^{\dagger}\left(\Pi-\widehat{\Pi}_{n}\right) A X+A^{\dagger}\left(\Pi-\widehat{\Pi}_{n}\right) \eta-A^{\dagger} \Pi \eta \\
& =A^{\dagger}\left(\left(\Pi-\widehat{\Pi}_{n}\right) Y-\Pi \eta\right),
\end{aligned}
$$

so that

$$
\|X-\widehat{X}\| \leq \frac{1}{\sigma_{d}}\left\|\left(\Pi-\widehat{\Pi}_{n}\right) Y-\Pi \eta\right\| .
$$

An application of (32) to the previous estimate gives the first bound of the statement. Similarly, we derive the second bound as follows. Equations (17), (7), and (34) yield

$$
\begin{aligned}
Q \eta-Q \widehat{\eta} & =A(X-\widehat{X}) \\
& =Q\left(\left(\Pi-\widehat{\Pi}_{n}\right) Y-\Pi \eta\right) .
\end{aligned}
$$

The other bounds follow by estimating them by multiples of $\|X-\widehat{X}\|$ as we show below. By definition of $\widehat{t}_{n}$

$$
\begin{aligned}
\left\|Z^{\widehat{t_{n}}}-X\right\| & \leq\left\|Z^{\widehat{t_{n}}}-\widehat{X}\right\|+\|X-\widehat{X}\| \\
& \leq\left\|Z^{t^{*}}-\widehat{X}\right\|+\|X-\widehat{X}\|, \\
& \leq\left\|Z^{t^{*}}-X\right\|+2\|X-\widehat{X}\| .
\end{aligned}
$$

Furthermore,

$$
\widehat{R}_{n}(t)-R(t)=X-\widehat{X}=A^{\dagger}\left(\left(\Pi-\widehat{\Pi}_{n}\right) Y-\Pi \eta\right),
$$

and triangle inequality gives

$$
\sup _{0 \leq t \leq 1}\left|\left\|\widehat{R}_{n}(t)\right\|-\|R(t)\|\right| \leq \sup _{0 \leq t \leq 1}\left\|\widehat{R}_{n}(t)-R(t)\right\|=\|X-\widehat{X}\| .
$$

All the remaining bounds in the statement of the theorem are now consequences of $(32)$. 
Remark 9 To justify and explain the consistency of the sampling strategy for approximation of the optimal regularization parameter $t^{*}$, let us assume that $n$ goes to infinity and $\sigma$ vanishes. Under this theoretical assumption, Theorem 8 shows that $\left\|\widehat{R}_{n}(t)\right\|$ convergences uniformly to $\|R(t)\|$ with high probability. The uniform convergence implies the $\Gamma$-convergence (see Braides, 2001), and, since the domain $[0,1]$ is compact, Theorem 1.22 in Braides (2001) ensures that

$$
\lim _{\substack{n \rightarrow+\infty \\ \sigma \rightarrow 0}}\left(\inf _{0 \leq t \leq 1}\left\|\widehat{R}_{n}(t)\right\|-\inf _{0 \leq t \leq 1}\|R(t)\|\right)=0 .
$$

While the compactness given by the $\Gamma$-convergence guarantees the consistency of the approximation to an optimal parameter, it is much harder for arbitrary $A$ to provide an error bound, depending on $n$. For the case $A=I$ in Section 4.4 we are able to establish very precise quantitative bounds with high probability.

Remark 10 Under the conditions of Theorem 8 for all $i=1, \ldots, d$ it holds as well

$$
\begin{aligned}
&\left|\xi_{i}-\widehat{\xi}_{i}\right| \lesssim \frac{1}{\sigma_{i}} B(n, \tau, \sigma) \\
&\left|\nu_{i}-\widehat{\nu_{i}}\right| \lesssim B(n, \tau, \sigma) .
\end{aligned}
$$

These bounds are a direct consequence of Theorem 8.

The following theorem is about the uniform approximation to the derivative function $H(t)$.

Theorem 11 Given $\tau>0$, with probability greater than $1-10 \mathrm{e}^{-\tau^{2}}$,

$$
\sup _{0 \leq t \leq 1}\left|\widehat{H}_{n}(t)-H(t)\right| \lesssim B(n, \tau, \sigma)\left(\frac{1}{\sigma_{p}^{3}}(\sqrt{h}+\tau)+\frac{\sigma}{\sigma_{d}^{4}}(\sqrt{d}+\tau)\right)
$$

provided that $n$ and $\sigma$ satisfy (27), where $p$ is defined in (9).

Proof Equations (22) and (35) give

$$
\begin{aligned}
\widehat{H}_{n}(t)-H(t) & =\left\langle\widehat{R}_{n}(t)-R(t), R^{\prime}(t)\right\rangle \\
& =\left\langle\left(\Pi-\widehat{\Pi}_{n}\right) Y-\Pi \eta,\left(A^{T}\right)^{\dagger} B(t)^{-2} A^{T} Y\right\rangle \\
& =\left\langle\left(\Pi-\widehat{\Pi}_{n}\right) Y-\Pi \eta,\left(t A A^{T}+(1-t) I\right)^{-2} Q Y\right\rangle,
\end{aligned}
$$

where we observe that $t A A^{T}+(1-t) I$ is invertible on $\operatorname{ran} Q$ and $A^{T} Y=A^{T} Q Y$. Hence,

$$
\begin{aligned}
\left|\widehat{H}_{n}(t)-H(t)\right| \leq & \left\|\left(\Pi-\widehat{\Pi}_{n}\right) Y-\Pi \eta\right\| \times \\
& \times\left(\left\|\left(t A A^{T}+(1-t) I\right)^{-2} A X\right\|+\sigma\left\|\left(t A A^{T}+(1-t) I\right)^{-2} Q W\right\|\right) .
\end{aligned}
$$

Furthermore, recalling that $A X=\Pi A X$ and $\Pi u_{i}=0$ for all $i>p,(31)$ implies that

$$
\begin{gathered}
\left\|\left(t A A^{T}+(1-t) I\right)^{-2} A X\right\| \leq \frac{\sigma_{p}}{\left(t \sigma_{p}^{2}+(1-t)\right)^{2}}\|X\| \lesssim \frac{1}{\sigma_{p}^{3}}(\sqrt{h}+\tau) \\
\left\|\left(t A A^{T}+(1-t) I\right)^{-2} Q W\right\| \leq \frac{1}{\left(t \sigma_{d}^{2}+(1-t)\right)^{2}}\|Q W\| \lesssim \frac{1}{\sigma_{d}^{4}}(\sqrt{d}+\tau)
\end{gathered}
$$


hold with probability greater than $1-4 \mathrm{e}^{-\tau^{2}}$. Bound (32) provides the desired claim.

The uniform approximation result of Theorem 11 allows us to claim that any $\widehat{t}_{n} \in[0,1]$ such that $\widehat{H}_{n}\left(\widehat{t}_{n}\right)=0$ can be attempted as a proxy for the optimal parameter $t^{*}$, especially if it is the only root in the interval $(0,1)$.

Nevertheless, being $\widehat{H}_{n}$ a sum of $d$ rational functions of polynomial numerator of degree 1 and polynomial denominator of degree 3 , the computation of its zeros in $[0,1]$ is equivalent to the computation of the roots of a polynomial of degree $3(d-1)+1=3 d-2$. The computation cannot be done analytically for $d>2$, because it would require the solution of a polynomial equation of degree larger than 4 . For $d>2$, we are forced to use numerical methods, but this is not a great deal as by now there are plenty of stable and reliable routines to perform such a task (for instance, Newton method, numerical computation of the eigenvalues of the companion matrix, just to mention a few).

We provide below relatively simple numerical experiments to validate the theoretical results reported above. In Figure 2 we show optimal parameters $t^{*}$ and corresponding approximations $\widehat{t}_{n}$ (computed by numerical solution to the scalar nonlinear equation $\widehat{H}_{n}(t)=0$ on $[0,1])$, for $n$ different data $Y=A X+\eta$. The accordance of the two parameters $t^{*}$ and $\widehat{t}_{n}$ is visually very convincing and their statistical (empirical) distributions reported in Figure 3 are also very close.

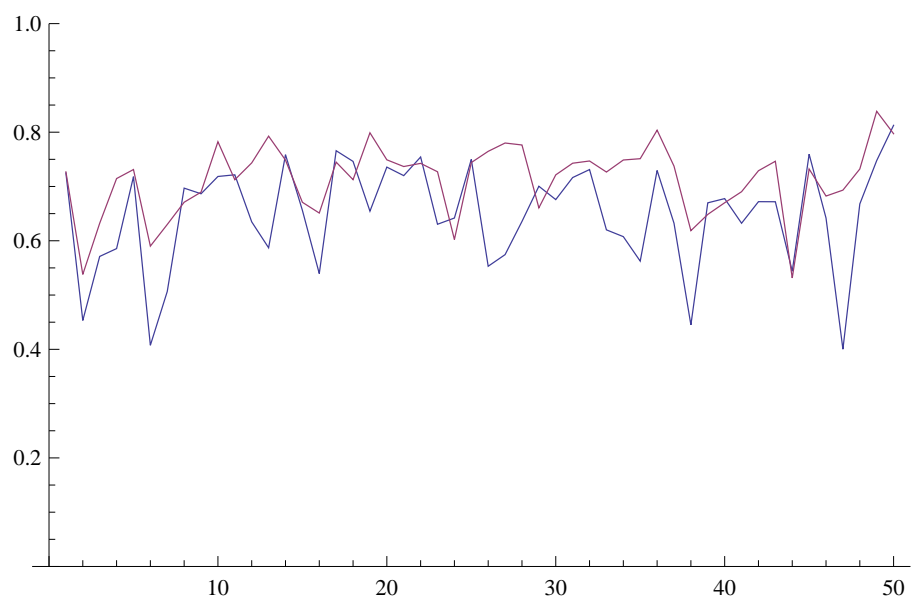

Figure 2: Optimal parameters $t^{*}$ and corresponding approximations $\widehat{t}_{n}(n=1000)$ for 50 different new data $Y=A X+\eta$ for $X \in \mathbb{R}^{d}$ and $\eta \in \mathbb{R}^{m}$ Gaussian vectors, $d=200$, $m=60$ and $A \in \mathbb{R}^{60 \times 200}$. Here we assumed that $X \in \mathcal{V}$ for $\mathcal{V}=\operatorname{span}\left\{\mathrm{e}_{1}, \ldots, \mathrm{e}_{5}\right\}$. We designed the matrix in such a way that the spectrum is vanishing, i.e., $\sigma_{\min } \approx$ 0 . Here we considered as noise level $\sigma=0.03$, so that the optimal parameter $t^{*}$ is rather concentrated around 0.7. The accordance of the two parameters $t^{*}$ and $\widehat{t}_{n}$ is visually very convincing.

In the next two sections we discuss special cases where we can provide even more precise statements and explicit bounds. 

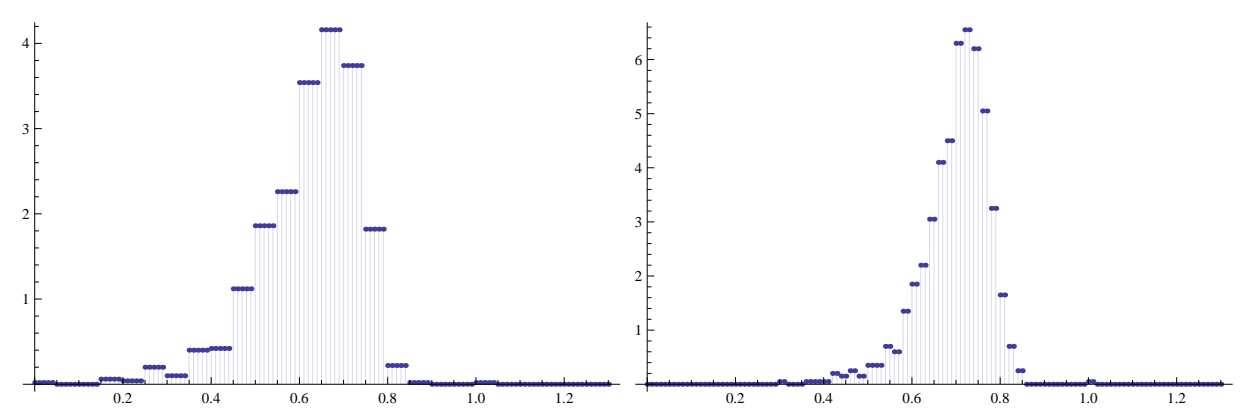

Figure 3: Empirical distribution of the optimal parameters $t^{*}$ (left) and the corresponding empirical distribution of the approximating parameters $\widehat{t}_{n}$ (right) for 1000 randomly generated data $Y=A X+\eta$ with the same noise level. The statistical accordance of the two parameters $t^{*}$ and $\widehat{t}_{n}$ is shown.

\section{Toy examples}

In the following we specify the results of the previous in simple cases, which allow to understand more precisely the behavior of the different estimators presented in Theorem 8 .

\subsection{Deterministic sparse signal and Bernoulli noise in two dimensions}

From Theorem 8 one might conclude that the estimators $\widehat{X}, Z^{t^{*}}, Z^{\widehat{t}_{n}}$ are all performing an approximation to $X$ with a least error proportional to $B(n, \tau, \sigma)$. With this in mind, one might be induced to conclude that computing $\widehat{X}$ is a sufficient effort, with no need of considering the empirical estimator $Z^{\widehat{t}_{n}}$, hence no need for computing $\widehat{t}_{n}$. In this section we discuss precisely this issue in some detail on a concrete toy example, for which explicit computations are easily performed.

Let us consider a deterministic sparse vector $X=(1,0)^{T} \in \mathbb{R}^{2}$ and a stochastic noise $W=\left(W_{1}, W_{2}\right)^{T} \in \mathbb{R}^{2}$ with Bernoulli entries, i.e., $W_{i}= \pm 1$ with probability $1 / 2$. This noise distribution is indeed Subgaussian with zero mean. For the sake of simplicity, we fix now an orthogonal matrix $A \in \mathbb{R}^{2 \times 2}$, which we express in terms of its vector-columns as follows: $A=\left(A_{1} \mid A_{2}\right)$. Notice that $\|W\|=\sqrt{2}$ with probability 1 and $\|X\|=1$. Hence, we may want to consider a noise level $\sigma \in[-1 / \sqrt{2}, 1 / \sqrt{2}]$. In view of the fact that the sign of $\sigma$ would simply produce a symmetrization of the signs of the component of the noise, we consider from now on only the cases where $W_{1} \geq 0$. The other cases are obtained simply by changing the sign of $\sigma$.

We are given measurements

$$
Y=A X+\sigma W
$$

While $X$ is deterministic (and this is just to simplify the computations) $Y$ is stochastic given the randomness of the noise. Hence, noticing that $Y=A X+\sigma W=A_{1}+\sigma W$ and observing that $\mathbb{E} W=0$, we obtain

$$
\Sigma_{Y}=\int Y Y^{T} d \mathbb{P}(W)=\int\left(A_{1}+\sigma W\right)\left(A_{1}+\sigma W\right)^{T} d \mathbb{P}(W)=A_{1} A_{1}^{T}+\sigma^{2} I .
$$


It is also convenient to write $\Sigma_{Y}$ by means of its complete singular value decomposition

$$
\Sigma_{Y}=\left(A_{1} A_{2}\right)\left(\begin{array}{ll}
1+\sigma^{2} & 0 \\
0 & \sigma^{2}
\end{array}\right)\left(\begin{array}{l}
A_{1}^{T} \\
A_{2}^{T}
\end{array}\right) .
$$

\subsection{Exact projection}

The projection onto the first largest singular space of $\Sigma_{Y}$ is simply given by

$$
\Pi \xi=\left\langle\xi, A_{1}\right\rangle A_{1} .
$$

This implies that

$$
\Pi Y=A_{1}+\sigma\left\langle W, A_{1}\right\rangle A_{1}
$$

From now on, we denote

$$
\sigma_{i}=\sigma\left\langle W, A_{i}\right\rangle, \quad i=1,2 .
$$

Let us stress that these parameters indicate how strong the particular noise instance $W$ is correlated with $\mathcal{W}=\operatorname{span}\left\{A_{1}=A X\right\}$. If $\left|\sigma_{1}\right|$ is large, then there is strong concentration of the particular noise instance on $\mathcal{W}$ and necessarily $\left|\sigma_{2}\right|$ is relatively small. If, vice versa, $\left|\sigma_{2}\right|$ is large, then there is poor correlation of the particular noise instance with $\mathcal{W}$.

We observe now that, being $A$ orthogonal, it is invertible and

$$
I=A^{T} A=A^{-1} A=\left(A^{-1} A_{1} A^{-1} A_{2}\right) .
$$

This means that $A^{-1} A_{1}=(1,0)^{T}$ and $A^{-1} A_{2}=(0,1)^{T}$ and we compute explicitly

$$
\bar{X}=A^{-1} \Pi Y=A^{-1} A_{1}+\sigma_{1} A^{-1} A_{1}=\left(1+\sigma_{1}, 0\right)^{T} .
$$

It is important to notice that for $\sigma_{1}$ small $\bar{X}$ is indeed a good proxy for $X$ and it does not belong to the set of possible Tikhonov regularizers, i.e., the minimizers of the functional

$$
t\|A Z-Y\|_{2}^{2}+(1-t)\|Z\|_{2}^{2}
$$

for some $t \in[0,1]$. This proxy $\bar{X}$ for $X$ approximates it with error exactly computed by

$$
\|\bar{X}-X\|=\left|\sigma_{1}\right| \leq \sqrt{2}|\sigma| .
$$

The minimizer of (36) is given by

$$
\begin{aligned}
Z^{t} & =t\left(t A^{T} A+(1-t) I\right)^{-1} A^{T} Y \\
& =t\left(t\left(A^{T}-A^{-1}\right) A+I\right)^{-1}\left(\begin{array}{c}
A_{1}^{T} \\
A_{2}^{T}
\end{array}\right)\left(A_{1}+\sigma W\right) .
\end{aligned}
$$

In view of the orthogonality of $A$ we have $A^{T}=A^{-1}$, whence $t\left(A^{T}-A^{-1}\right) A=0$, and the simplification

$$
\begin{aligned}
Z^{t} & =t\left(\begin{array}{c}
A_{1}^{T} \\
A_{2}^{T}
\end{array}\right)\left(A_{1}+\sigma W\right) \\
& =t\left(1+\sigma_{1}, \sigma_{2}\right)^{T}
\end{aligned}
$$


Now it is not difficult to compute

$$
R(t)^{2}=\left\|Z^{t}-X\right\|_{2}^{2}=\left(t\left(1+\sigma_{1}\right)-1\right)^{2}+t^{2} \sigma_{2}^{2}=\left(\left(1+\sigma_{1}\right)^{2}+\sigma_{2}^{2}\right) t^{2}-2 t\left(1+\sigma_{1}\right)+1
$$

This quantity is optimized for

$$
t^{*}=\frac{1+\sigma_{1}}{\left(1+\sigma_{1}\right)^{2}+\sigma_{2}^{2}}
$$

The direct substitution gives

$$
R\left(t^{*}\right)=\frac{\left|\sigma_{2}\right|}{\sqrt{\left(1+\sigma_{1}\right)^{2}+\sigma_{2}^{2}}}
$$

And now we notice that, according to (37) one can have either

$$
\|\bar{X}-X\|=\left|\sigma_{1}\right|<\frac{\left|\sigma_{2}\right|}{\sqrt{\left(1+\sigma_{1}\right)^{2}+\sigma_{2}^{2}}}=\left\|Z^{t^{*}}-X\right\|
$$

or

$$
\|\bar{X}-X\|=\left|\sigma_{1}\right|>\frac{\left|\sigma_{2}\right|}{\sqrt{\left(1+\sigma_{1}\right)^{2}+\sigma_{2}^{2}}}=\left\|Z^{t^{*}}-X\right\|
$$

very much depending on $\sigma_{i}=\sigma\left\langle W, A_{i}\right\rangle$. Hence, for the fact that $\bar{X}$ does not belong to the set of minimizers of (36), it can perfectly happen that it is a better proxy of $X$ than $Z^{t^{*}}$.

Recalling that $\bar{X}=\left(1+\sigma_{1}, 0\right)^{T}$ and $Z^{t}=t\left(1+\sigma_{1}, \sigma_{2}\right)^{T}$, let us now consider the error

$$
\begin{aligned}
\bar{R}(t)^{2} & =\left\|Z^{t}-\bar{X}\right\|^{2} \\
& =\left(\left(1+\sigma_{1}\right)^{2}+\sigma_{2}^{2}\right) t^{2}-2 t\left(1+\sigma_{1}\right)^{2}+\left(1+\sigma_{1}\right)^{2} .
\end{aligned}
$$

This is now opimized for

$$
\bar{t}=\frac{\left(1+\sigma_{1}\right)^{2}}{\left(1+\sigma_{1}\right)^{2}+\sigma_{2}^{2}}
$$

Hence we have

$$
R(\bar{t})^{2}=\left\|Z^{\bar{t}}-X\right\|^{2}=\left(\left(1+\sigma_{1}\right)^{2}+\sigma_{2}^{2}\right) \bar{t}^{2}-2 \bar{t}\left(1+\sigma_{1}\right)+1=\frac{\sigma_{1}^{2}\left(1+\sigma_{1}\right)^{2}+\sigma_{2}^{2}}{\left(1+\sigma_{1}\right)^{2}+\sigma_{2}^{2}}
$$

or

$$
R(\bar{t})=\frac{\sqrt{\sigma_{1}^{2}\left(1+\sigma_{1}\right)^{2}+\sigma_{2}^{2}}}{\sqrt{\left(1+\sigma_{1}\right)^{2}+\sigma_{2}^{2}}}
$$

In this case we notice that, in view of the fact that $\sigma_{1}^{2} \leq 1$ for $|\sigma| \leq 1 / \sqrt{2}$ one can have only

$$
\|\bar{X}-X\|=\left|\sigma_{1}\right| \leq \frac{\sqrt{\sigma_{1}^{2}\left(1+\sigma_{1}\right)^{2}+\sigma_{2}^{2}}}{\sqrt{\left(1+\sigma_{1}\right)^{2}+\sigma_{2}^{2}}}=\left|\sigma_{1}\right| \frac{\sqrt{\left(1+\sigma_{1}\right)^{2}+\sigma_{2}^{2} / \sigma_{1}^{2}}}{\sqrt{\left(1+\sigma_{1}\right)^{2}+\sigma_{2}^{2}}}=\left\|Z^{\bar{t}}-X\right\|
$$

independently of $\sigma_{i}=\sigma\left\langle W, A_{i}\right\rangle$. The only way of reversing the inequality is by allowing noise level $\sigma$ such that $\sigma_{1}^{2}>1$, which would mean that we have noise significantly larger than the signal. 


\subsubsection{Concrete EXAMPles}

Let us make a few concrete examples. Let us recall our assumpution that $\operatorname{sign} W_{1}=+1$ while the one of $\mathrm{W}_{2}$ is kept arbitrary. Suppose that $A_{1}=\frac{W}{\|W\|}$, then $\sigma_{1}=\sigma \sqrt{2}$ and $\sigma_{2}=0$. In this case we get

$$
\sigma \sqrt{2}=\|\bar{X}-X\|=\left\|Z^{\bar{t}}-X\right\|
$$

and $\bar{X}=Z^{\bar{t}}$. The other extreme case is given by $A_{2}=\frac{W}{\|W\|}$, then $\sigma_{1}=0$ and $\sigma_{2}= \pm \sigma \sqrt{2}$. In this case $\bar{X}=X$, while $R(\bar{t})=R\left(t^{*}\right)=|\sigma| \sqrt{2 /\left(1+2 \sigma^{2}\right)}>0$. An intermediate case is given precisely by $A=I$, which we shall investigate in more generality in Section 4.4. For this choice we have $\sigma_{1}=\sigma$ and $\sigma_{2}= \pm \sigma$. Notice that the sign of $\sigma_{2}$ does not matter in the expressions of $R(\bar{t})$ and $R\left(t^{*}\right)$ because it appears always squared. Instead the sign of $\sigma_{1}$ matters and depends on the choice of the sign of $\sigma$. We obtain

$$
R\left(t^{*}\right)=\frac{|\sigma|}{\sqrt{(1+\sigma)^{2}+\sigma^{2}}}
$$

and

$$
R(\bar{t})=\sqrt{\frac{\sigma^{2}(2+(\sigma(2+\sigma))}{1+2 \sigma(1+\sigma)}},
$$

and, of course

$$
\|\bar{X}-X\|=|\sigma|
$$

In this case, $R\left(t^{*}\right) \leq\|\bar{X}-X\|$ if and only if $\sigma \geq 0$, while - unfortunately $-R(\bar{t})>\|\bar{X}-X\|$ for all $|\sigma|<1$ and the inequality gets reversed only if $|\sigma| \geq 1$.

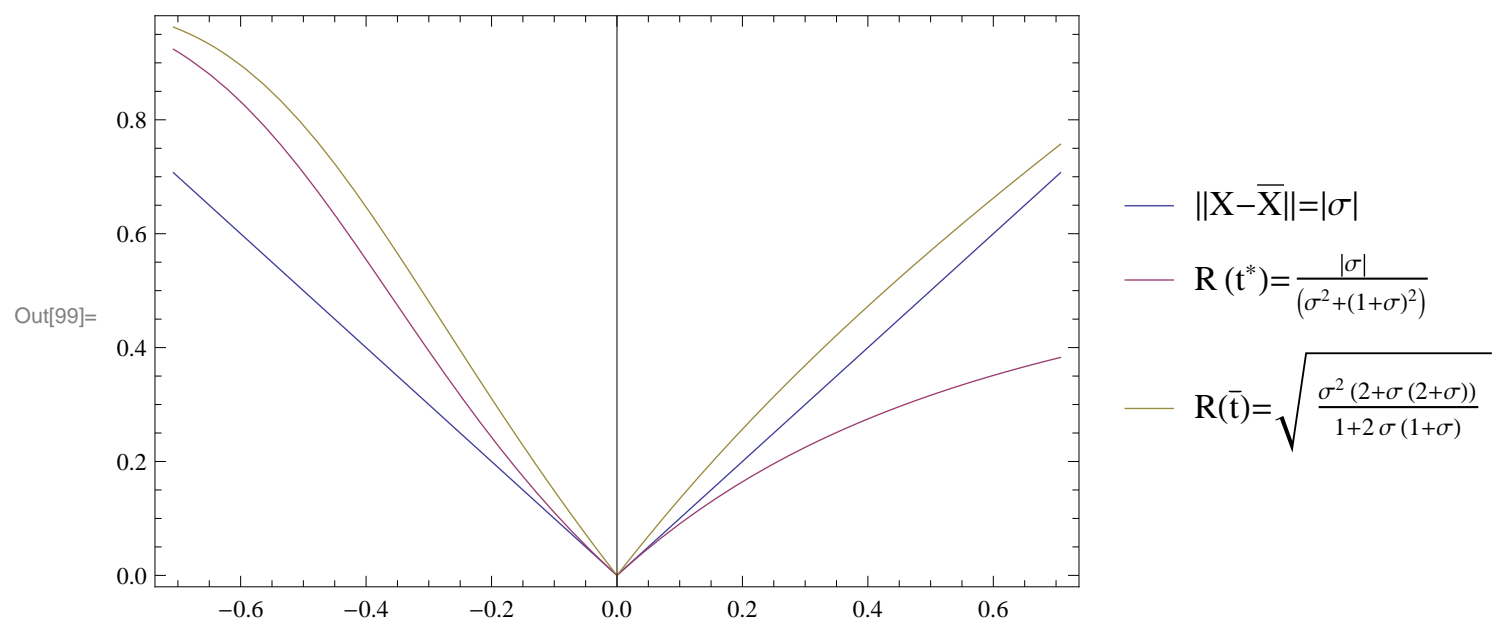

Figure 4: Comparison of $\|\bar{X}-X\|, R\left(t^{*}\right)$, and $R(\bar{t})$ as a function of $\sigma$ for $\sigma_{1}=\sigma$.

In Figure 5 we illustrate the case of $\sigma_{1}=1.2 \sigma$ and in Figure 6 the case of $\sigma_{1}=0.7 \sigma$. Let us now focus for a moment on the purely denoising case $A=I$. In this case, the projection $\Pi$ is actually the projection onto the subspace where $X$ is defined. While $\bar{X}$ belongs also to 


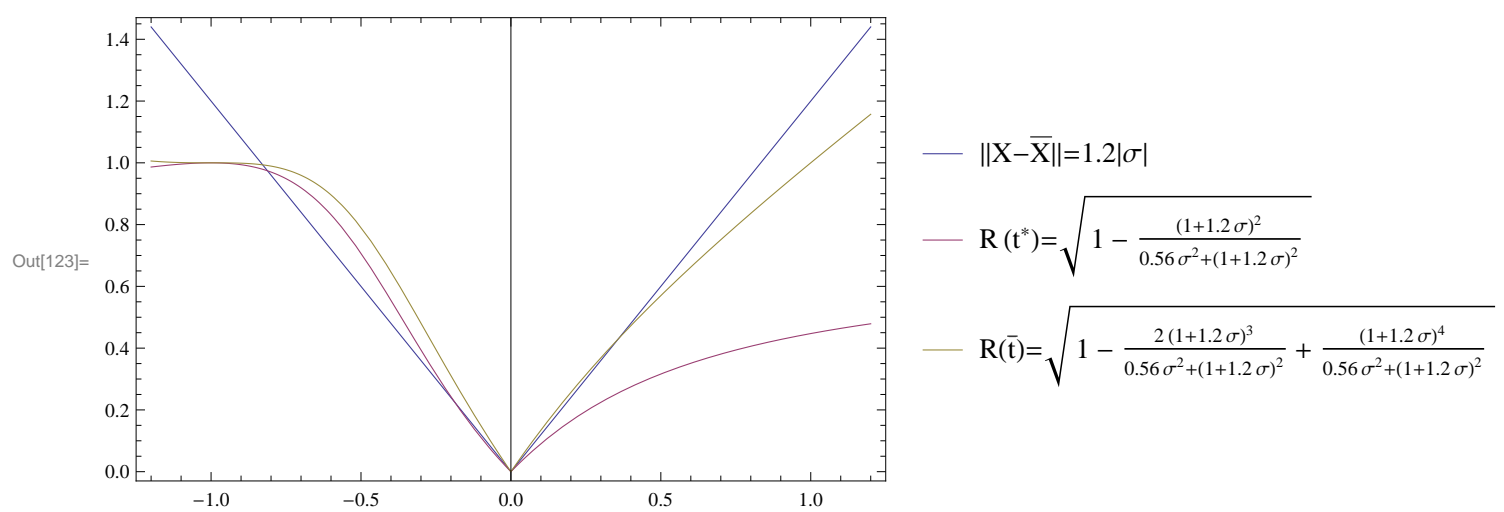

Figure 5: Comparison of $\|\bar{X}-X\|, R\left(t^{*}\right)$, and $R(\bar{t})$ as a function of $\sigma$ for $\sigma_{1}=1.2 \sigma$.

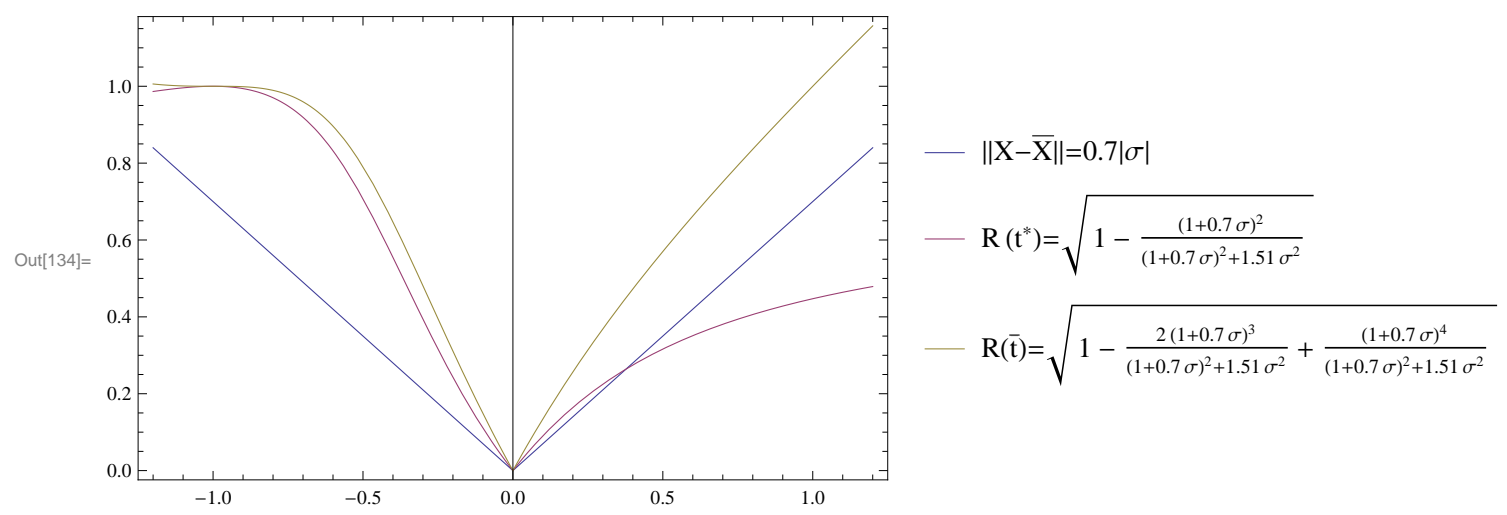

Figure 6: Comparison of $\|\bar{X}-X\|, R\left(t^{*}\right)$, and $R(\bar{t})$ as a function of $\sigma$ for $\sigma_{1}=0.7 \sigma$.

that subspace, this is not true for $Z^{t}$ in general. Since we do dispose of (an approximation) to $\Pi$ when $A=I$, it would be better to compare

$$
\|\bar{X}-X\|,\left\|\Pi Z^{t^{*}}-X\right\|,\left\|\Pi Z^{\bar{t}}-X\right\|
$$

as functions of $\sigma$. In general, we have

$$
R^{\Pi}(t)^{2}=\left\|\Pi Z^{t}-X\right\|^{2}=\left(1+\sigma_{1}\right)^{2} t^{2}-2 t\left(1+\sigma_{1}\right)+1
$$

and

$$
\begin{aligned}
& R^{\Pi}(\bar{t})=\frac{\left(\sigma_{2}^{2}-\sigma_{1}\left(\sigma_{1}+1\right)^{2}\right)}{\left(\sigma_{2}^{2}+\left(\sigma_{2}+1\right)^{2}\right)}, \\
& R^{\Pi}\left(t^{*}\right)=\frac{\sigma_{2}^{2}}{\left(\sigma_{2}^{2}+\left(\sigma_{1}+1\right)^{2}\right)} .
\end{aligned}
$$

The comparison of these quantities is reported in Figure 7 and one can observe that, at least for $\sigma \geq 0$, both $\Pi Z^{t^{*}}$ and $\Pi Z^{\bar{t}}$ are better approximations of $X$ than $\bar{X}$. 


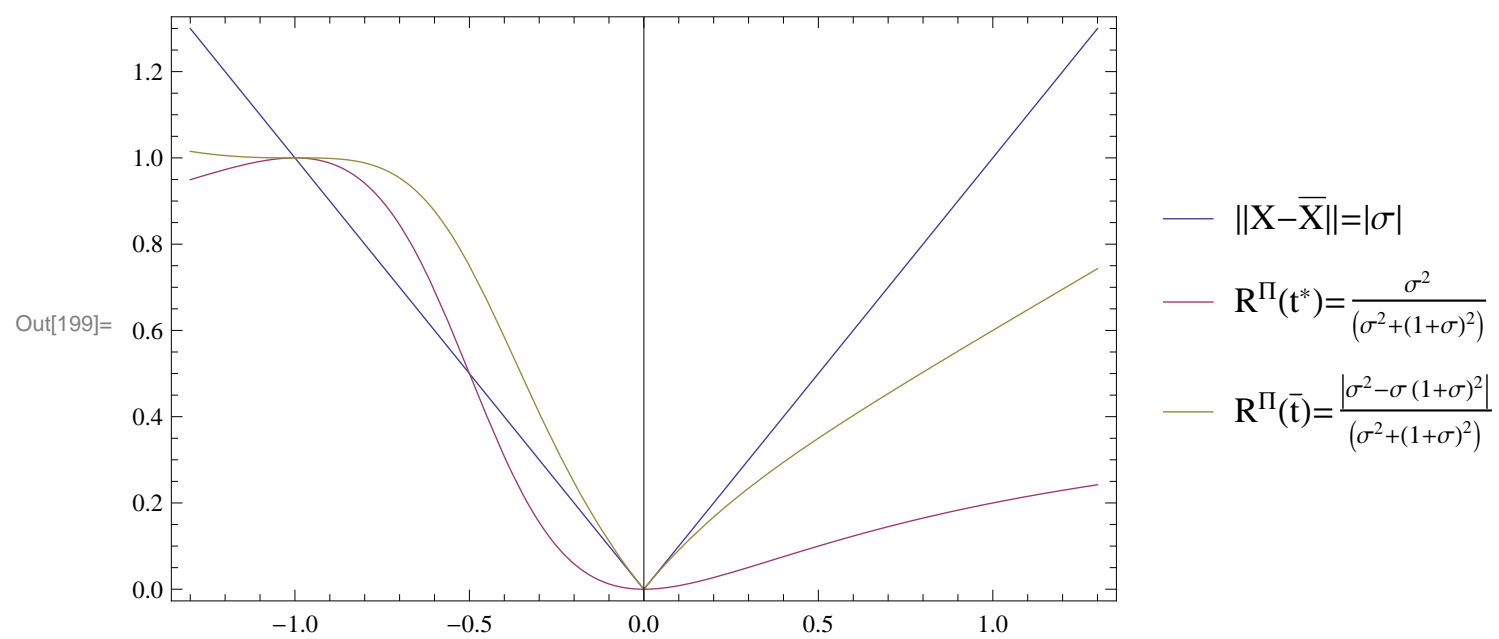

Figure 7: Comparison of $\|\bar{X}-X\|, R^{\Pi}(\bar{t})$, and $R\left(t^{*}\right)$ as a function of $\sigma$ for $\sigma_{1}=\sigma$.

\subsection{Empirical projections}

In concrete applications we do not dispose of $\Pi$ but only of its empirical proxy $\widehat{\Pi}_{n}$. According to Theorem 4 they are related by the approximation

$$
\left\|\Pi-\widehat{\Pi}_{n}\right\| \leq \frac{1}{\lambda_{\min }}\left(\sqrt{\frac{m}{n}}+\frac{\tau}{\sqrt{n}}\right),
$$

with high probability (depending on $\tau>0$ ), where $m=1$ in our example and $\lambda_{\min }=1$ is the smallest nonzero eigenvalue of $\Sigma_{A X}=A_{1} A_{1}^{T}$. Hence, we compute

$$
\widehat{X}=A^{-1} \widehat{\Pi}_{n} Y
$$

and, since $A$ is an orthogonal matrix

$$
\begin{aligned}
\|X-\widehat{X}\| & =\left\|A^{-1}\left(\Pi-\widehat{\Pi}_{n}\right) Y\right\| \\
& =\left\|\left(\Pi-\widehat{\Pi}_{n}\right) Y\right\| \\
& \leq \frac{1+\tau}{\sqrt{n}}\left\|A_{1}+\sigma W\right\| \\
& \leq \frac{1+\tau}{\sqrt{n}} \sqrt{1+2 \sigma^{2}+2 \sigma_{1}} \leq \frac{1+\tau}{\sqrt{n}}(1+\sqrt{2} \sigma) .
\end{aligned}
$$

Here we used

$$
\left\|A_{1}+\sigma W\right\|^{2}=\left\|A_{1}\right\|^{2}++\sigma^{2}\|W\|^{2}+2 \sigma\left\langle A_{1}, W\right\rangle=1+2 \sigma^{2}+2 \sigma_{1} .
$$

In view of the approximation relationship above, we can express now

$$
\widehat{X}=\left(1+\sigma_{1}+\epsilon_{1}, \epsilon_{2}\right)^{T},
$$

where $\sqrt{\epsilon_{1}^{2}+\epsilon_{2}^{2}} \leq \frac{1+\tau}{\sqrt{n}}(1+\sqrt{2} \sigma)$. Then

$$
\|X-\widehat{X}\|=\sqrt{\left(\sigma_{1}+\epsilon_{1}\right)^{2}+\epsilon_{2}^{2}}
$$


One has also

$$
\begin{aligned}
R_{n}(t)^{2}=\left\|Z^{t}-\widehat{X}\right\|^{2} & =\left(t\left(1+\sigma_{1}\right)-\left(1+\sigma_{1}+\epsilon_{1}\right)\right)^{2}+\left(t \sigma_{2}-\epsilon_{2}\right)^{2} \\
& =t^{2}\left(\left(1+\sigma_{1}\right)^{2}+\sigma_{2}^{2}\right)-2 t\left(\sigma_{2} \epsilon_{2}+\left(1+\sigma_{1}\right) \epsilon_{1}+\left(1+\epsilon_{1}\right)^{2}\right)+\text { const. }
\end{aligned}
$$

Hence, its optimizer is given by

$$
\widehat{t}_{n}=\frac{\left(\sigma_{2} \epsilon_{2}+\left(1+\sigma_{1}\right) \epsilon_{1}+\left(1+\epsilon_{1}\right)^{2}\right)}{\left(1+\sigma_{1}\right)^{2}+\sigma_{2}^{2}}
$$

and

$$
R\left(\widehat{t}_{n}\right)=\left\|Z^{\widehat{t}_{n}}-X\right\|=\sqrt{\frac{\left(\sigma_{2}^{2}\left(1+\epsilon_{2}\right)^{2}+2 \epsilon_{2}\left(1+\sigma_{1}\right)\left(\epsilon_{1}+\sigma_{1}\right) \sigma_{2}+\left(1+\sigma_{1}\right)^{2}\left(\epsilon_{1}+\sigma_{1}\right)^{2}\right)}{\left(1+\sigma_{1}\right)^{2}+\sigma_{2}^{2}}}
$$

\subsubsection{CONCRETE EXAMPle}

We compare in Figure 8 the behavior of the difference of the errors

$$
\|X-\widehat{X}\|-\left\|Z^{\widehat{t}_{n}}-X\right\|
$$

depending on $\sigma$, for $\sigma_{1}=1.3 \sigma$ and $n=100$. In this case, the empirical estimator $Z^{\widehat{t}_{n}}$ is a significantly better approximation to $X$ than $\widehat{X}$ for all noise levels $\sigma \in[-0.07,1 / \sqrt{2}]$, while $\widehat{X}$ keeps being best estimator, e.g., for $\sigma \in[-1 / \sqrt{2},-0.1]$.

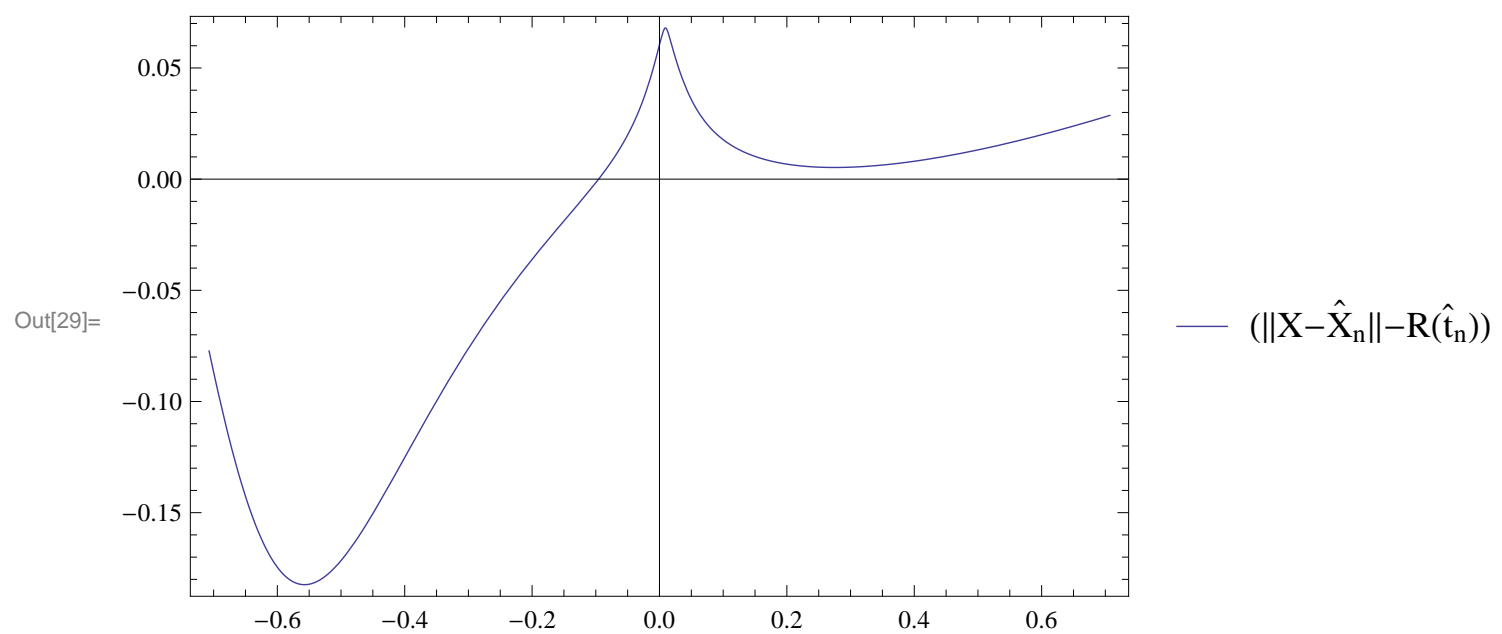

Figure 8: The difference of the errors $\|X-\widehat{X}\|-\left\|Z^{\widehat{t}_{n}}-X\right\|$ as a function of $\sigma$ for $\sigma_{1}=1.3 \sigma$. For $\sigma \in[-0.07,1 / \sqrt{2}]$ one has $\|X-\widehat{X}\|>\left\|Z^{\widehat{t}_{n}}-X\right\|$.

From these simple two dimensional toy examples and related numerical experiments we deduce the following general principles:

- The performances of the estimators $\bar{X}, Z^{t^{*}}, Z^{\widehat{t}_{n}}$ depend very much on how the noise is distributed with respect to the subspace $\mathcal{W}$ to which $A X$ belongs and its signature; 
if the noise is not very much concentrated on such subspace, then $\bar{X}$ tends to be a better estimator, see Fig. 6 , while a concentrated noise would make $\bar{X}, Z^{\widehat{t}_{n}}$ rather equivalent and $Z^{t^{*}}$ the best one for some ranges of noise, see Fig. 4 and Fig. 5.

- If the number $n$ of samples is not very large (in the example above we considered $n=100)$, so that the approximation $A^{-1} \widehat{\Pi}_{n} Y=\widehat{X} \approx \bar{X}=A^{-1} \Pi Y$ would be still too rough, then the estimator $Z^{\widehat{t}_{n}}$ may beat $\widehat{X}$ for significant ranges of noise, independently of its instance correlation with $\mathcal{W}$, see Fig. 8 .

Since it is a priori impossible to know precisely in which situation $\widehat{X}, Z^{\widehat{t}_{n}}$ perform better as estimators (as it depends on the correlation with $\mathcal{W}$ of the particular noice instance), in the practice it will be convenient to compute them both, especially when one does not dispose of a large number $n$ of samples.

\subsection{The case $A=I$}

As yet one more example, this time in higher dimension, we consider the simple case where $m=d$ and $A=I$, so that $\mathcal{W}=\mathcal{V}$. In this case, we get that

$$
R(t)=-(1-t) X+t \eta \text {. }
$$

If $Y \neq 0$, an easy computation shows that the minimizer of the reconstruction error $\|R(t)\|^{2}$ is

$$
t^{*}=t^{*}(Y, X)=\varphi\left(\frac{\langle Y, X\rangle}{\langle Y, Y\rangle}\right)
$$

where

$$
\varphi(s)= \begin{cases}0 & \text { if } s \leq 0 \\ s & \text { if } 0<s<1 \\ 1 & \text { if } s \geq 1\end{cases}
$$

If $Y=0$, the solution $Z^{t}$ does not depend on $t$, so that there is not a unique optimal parameter and we set $t^{*}=0$.

We further assume that $X$ is bounded from 0 with high probability, more precisely,

$$
\mathbb{P}[\|X\|<r] \leq 2 \exp \left(-\frac{1}{r^{2}}\right) .
$$

This assumption is necessary to avoid that the noise is much bigger than the signal.

Theorem 12 Given $\tau \geq 1$, with probability greater than $1-6 \mathrm{e}^{-\tau^{2}}$

$$
\left|\widehat{t}_{n}-t^{*}\right| \leq \frac{1}{\lambda_{\min }}\left(\sqrt{\frac{d}{n}}+\frac{\tau}{\sqrt{n}}+\sigma^{2}\right)+\sigma \ln \left(\frac{\mathrm{e}}{\sigma}\right)(\sqrt{h}+\tau),
$$

provided that

$$
\begin{aligned}
& n \gtrsim(\sqrt{d}+\tau)^{2} \max \left\{\frac{64}{\lambda_{\min }^{2}}, 1\right\} \\
& \sigma<\min \left\{\sqrt{\frac{\lambda_{\min }}{8}}, \mathrm{e}^{1-16 \tau^{2}}\right\} .
\end{aligned}
$$


Proof Without loss of generality, we assume that $\lambda_{\min } \leq 8$. Furthermore, on the event $\{Y=0\}$, by definition $t^{*}=\widehat{t}_{n}=0$, so that we can further assume that $Y \neq 0$.

Since $\varphi$ is a Lipschitz continuous function with Lipschitz constant 1 ,

$$
\begin{aligned}
\left|\widehat{t}_{n}-t^{*}\right| & \leq \frac{|\langle Y, \widehat{X}-X\rangle|}{\|Y\|^{2}} \\
& \leq \frac{\left\|\left(\Pi-\widehat{\Pi}_{n}\right) Y-\Pi \eta\right\|}{\|Y\|} \\
& \leq\left\|\left(\Pi-\widehat{\Pi}_{n}\right)\right\|+\sigma \frac{\|\Pi W\|}{\|Y\|},
\end{aligned}
$$

where the second inequality is consequence of (34). Since (41a) and (41b) imply (27) and $m=d$, by $(26)$ we get

$$
\left\|\widehat{\Pi}_{n}-\Pi\right\| \lesssim \frac{1}{\lambda_{\min }}\left(\sqrt{\frac{d}{n}}+\frac{\tau}{\sqrt{n}}+\sigma^{2}\right)
$$

with probability greater than $1-2 \mathrm{e}^{-\tau^{2}}$. It is now convenient to denote the probability distribution of $X$ as $\rho_{X}$, i.e., $X \sim \rho_{X}$. Fixed $r>0$, set

$$
\Omega=\{\|X\|<r\} \cup\left\{2 \sigma\langle X, W\rangle<-\|X\|^{2} / 2\right\},
$$

whose probability is bounded by

$$
\begin{aligned}
\mathbb{P}[\Omega] & \leq \mathbb{P}[\|X\|<r]+\mathbb{P}\left[4 \sigma\langle X, W\rangle<-\|X\|^{2},\|X\| \geq r\right] \\
& =\mathbb{P}[\|X\|<r]+\int_{\|x\| \geq r} \mathbb{P}\left[4 \sigma\langle x, W\rangle<-\|x\|^{2}\right] d \rho_{X}(x) \\
& \leq \mathbb{P}[\|X\|<r]+\int_{\|x\| \geq r} \exp \left(-\frac{\|x\|^{2}}{256 \sigma^{2}}\right) d \rho_{X}(x) \\
& \leq \mathbb{P}[\|X\|<r]+\exp \left(-\frac{r^{2}}{256 \sigma^{2}}\right),
\end{aligned}
$$

where we use (46c) with $\xi=-W$ (and the fact that $W$ and $X$ are independent), $\tau=$ $\|x\| /(16 \sigma)$ and $\|W\|_{\psi_{2}}=1 / \sqrt{2}$. With the choice $r=16 \tau / \ln (\mathrm{e} / \sigma)$, we obtain

$$
\begin{aligned}
\mathbb{P}[\Omega] & \leq \mathbb{P}[\|X\|<16 \tau / \ln (\mathrm{e} / \sigma)]+\exp \left(-\frac{\tau^{2}}{\sigma^{2} \ln ^{2}(\mathrm{e} / \sigma)}\right) \\
& \leq \mathbb{P}[\|X\|<16 \tau / \ln (\mathrm{e} / \sigma)]+\exp \left(-\tau^{2}\right),
\end{aligned}
$$

where $\sigma \mapsto \sigma \ln (\mathrm{e} / \sigma)$ is an increasing positive function on $(0,1]$, so that it is bounded by 1 . Furthermore, by (41b), i.e., $16 \tau / \ln (\mathrm{e} / \sigma) \leq \frac{1}{\tau}$, we have

$$
\mathbb{P}[\|X\|<16 \tau / \ln (\mathrm{e} / \sigma)] \leq P\left[\|X\|<\frac{1}{\tau}\right] \leq 2 \exp \left(-\tau^{2}\right),
$$


by Assumption (39). Hence it holds that $\mathbb{P}[\Omega] \leq 3 e^{-\tau^{2}}$.

On the event $\Omega^{c}$

$$
\begin{aligned}
\|Y\|^{2} & =\|X\|^{2}+2 \sigma\langle X, W\rangle+\sigma^{2}\|W\|^{2} \\
& \geq\|X\|^{2}+2 \sigma\langle X, W\rangle \geq\|X\|^{2} / 2 \\
& \geq r^{2} / 2 \simeq \tau^{2} / \ln ^{2}(\mathrm{e} / \sigma) \geq 1 / \ln ^{2}(\mathrm{e} / \sigma)
\end{aligned}
$$

since $\tau \geq 1$. Finally, (51) with $\xi=\Pi W \in \mathcal{W}$ yields

$$
\|\Pi W\| \lesssim(\sqrt{h}+\tau)
$$

with probability greater than $1-\exp \left(-\tau^{2}\right)$. Taking into account the above estimates, we conclude with probability greater than $1-4 \exp \left(-\tau^{2}\right)$ that

$$
\frac{\|\Pi W\|}{\|Y\|} \lesssim \ln \frac{\mathrm{e}}{\sigma}(\sqrt{h}+\tau)
$$

Then, with probability greater than $1-6 \exp \left(-\tau^{2}\right)$, we conclude the estimate

$$
\left|\widehat{t}_{n}-t^{*}\right| \lesssim \frac{1}{\lambda_{\min }}\left(\sqrt{\frac{d}{n}}+\frac{\tau}{\sqrt{n}}+\sigma^{2}\right)+\sigma \ln (\mathrm{e} / \sigma)(\sqrt{h}+\tau) .
$$

Remark 13 The function $\ln (\mathrm{e} / \sigma)$ can be replaced by any positive function $f(\sigma)$ such that $\sigma f(\sigma)$ is an infinitesimal function bounded by 1 in the interval $(0,1]$. The condition (41b) becomes

$$
\sigma<\min \left\{\sqrt{\frac{\lambda_{\min }}{8}}, 1\right\} \quad f(\sigma) \geq 16 \tau^{2},
$$

and, if $f$ is strictly decreasing,

$$
\sigma<\min \left\{\sqrt{\frac{\lambda_{\min }}{8}}, 1, f^{-1}\left(16 \tau^{2}\right)\right\} .
$$

Theorem 12 shows that if the number $n$ of examples is large enough and the noise level is small enough, the estimator $\widehat{t}_{n}$ is a good approximation of the optimal value $t^{*}$. Let us stress very much that the number $n$ of samples needed to achieve a good accuracy depends at most algebraically on the dimension $d$, more precisely $n=\mathcal{O}(d)$. Hence, in this case one does not incur in the curse of dimensionality. Moreover, the second term of the error estimate (40) gets smaller for smaller dimensionality $h$.

Remark 14 If there exists an orthonormal basis $\left(e_{i}\right)_{i}$ of $\mathbb{R}^{d}$, such that the random variables $\left\langle W, e_{1}\right\rangle, \ldots,\left\langle W, e_{d}\right\rangle$ are independent with $\mathbb{E}\left[\left\langle W, e_{1}\right\rangle^{2}\right]=1$, then Rudelson and Vershynin 
(2015, Theorem 2.1) showed that $\|W\|$ concentrates around $\sqrt{d}$ with high probability. Reasoning as in the proof of Theorem 12, by replacing $\|X\|^{2}$ with $\sigma^{2}\|W\|^{2}$, with high probability it holds that

$$
\left|\widehat{t}_{n}-t^{*}\right| \lesssim \frac{1}{\lambda_{\min }}\left(\sqrt{\frac{d}{n}}+\frac{\tau}{\sqrt{n}}+\sigma^{2}\right)+\frac{1}{\sqrt{d}}(\sqrt{h}+\tau) \frac{\tau}{\sqrt{d}}
$$

without assuming condition (39).

In Figures 9-12, we show examples of numerical accordance between optimal and estimated regularization parameters. In this case, the agreement between optimal parameter $t^{*}$ and learned parameter $\widehat{t}_{n}$ is overwhelming.



Figure 9: Optimal parameters $t^{*}$ and corresponding approximations $\widehat{t}_{n}(n=1000)$ for 50 different data $Y=X+\eta$ for $X$ and $\eta$ generated randomly with Gaussian distributions in $\mathbb{R}^{d}$ for $d=1000$. We assume that $X \in \mathcal{V}$ for $\mathcal{V}=\operatorname{span}\left\{\mathrm{e}_{1}, \ldots, \mathrm{e}_{5}\right\}$.
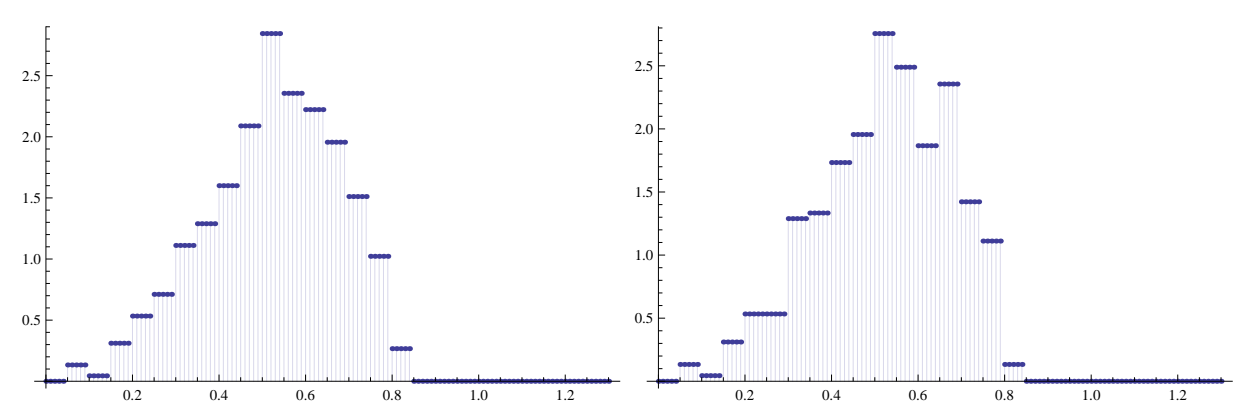

Figure 10: Empirical distribution of the optimal parameters $t^{*}$ (left) and the corresponding empirical distribution of the learned parameters $\widehat{t}_{n}$ (right) for 500 randomly generated data $Y=X+\eta$ with the same noise level. 


\section{An explicit formula by linearization}

While it is not possible to solve the equation $H(t) \approx \widehat{H}_{n}(t)=0$ by analytic methods for $d>2$ in the general case, one might attempt a linearization of this equation in certain regimes. It is well-known that the optimal Tikhonov regularization parameter $\alpha^{*}=\left(1-t^{*}\right) / t^{*}$ converges to 0 for vanishing noise level and this means that $t^{*}=t^{*}(\sigma) \rightarrow 1$ as $\sigma \rightarrow 0$. Hence, if the matrix $A$ has a significant spectral gap, i.e., $\sigma_{1} \geq \sigma_{2} \geq \cdots \geq \sigma_{d} \gg 0$ and $\sigma \approx 0$ is small enough, then

$$
\sigma_{i} \gg\left(1-t^{*}\right)
$$

and in this case

$$
\widehat{h}_{i}(t)=\frac{\left(\sigma_{i} \widehat{\nu}_{i} \widehat{\xi}_{i}^{-1}+1\right) t-1}{\left((1-t)+t \sigma_{i}^{2}\right)^{3}} \approx \frac{\left(\sigma_{i} \widehat{\nu}_{i} \widehat{\xi}_{i}^{-1}+1\right) t-1}{\sigma_{i}^{6}}, \quad t \approx t^{*} .
$$

The above linear approximation is equivalent to replacing $B(t)^{-1}$ with $B(1)^{-1}=\left(A^{T} A\right)^{-1}$ and Equation (23) is replaced by the following proxy (at least if $t \approx t^{*}$ )

$$
\begin{aligned}
\widehat{H}_{n}^{\operatorname{lin}}(t) & =\left\langle t A A^{T}\left(Y-\widehat{\Pi}_{n} Y\right)-(1-t) \widehat{\Pi}_{n} Y,\left(A A^{T}\right)^{\dagger 3} Y\right\rangle \\
& \left.=\left\langle A\left(A^{T} A\right)^{-3}\left(-(1-t) \widehat{X}+t A^{T} \widehat{\eta}\right), A \widehat{X}+\widehat{\eta}\right)\right\rangle \\
& =\left(\left\langle A\left(A^{T} A\right)^{-3}\left(\widehat{X}+A^{T} \widehat{\eta}\right), A \widehat{X}+\widehat{\eta}\right\rangle\right) t-\left\langle A\left(A^{T} A\right)^{-3} \widehat{X}, A \widehat{X}+\widehat{\eta}\right\rangle \\
& =\left(\sum_{i=1}^{d} \frac{\widehat{\alpha}_{i}}{\sigma_{i}^{5}}\left(\sigma_{i} \widehat{\nu}_{i} \widehat{\xi}_{i}^{-1}+1\right)\right) t-\sum_{i=1}^{d} \frac{\widehat{\alpha}_{i}}{\sigma_{i}^{5}}
\end{aligned}
$$

The only zero of $\widehat{H}_{n}^{\operatorname{lin}}(t)$ is

$$
\begin{aligned}
\widehat{t}_{n}^{\text {in }} & =\frac{\left\langle\widehat{\Pi}_{n} Y,\left(A A^{T}\right)^{\dagger 3} Y\right\rangle}{\left\langle A A^{T}\left(Y-\widehat{\Pi}_{n} Y\right)+\widehat{\Pi}_{n} Y,\left(A A^{T}\right)^{\dagger 3} Y\right\rangle} \\
& =1-\frac{\left\langle Y-\widehat{\Pi}_{n} Y,\left(A A^{T}\right)^{\dagger 2} Y\right\rangle}{\left\langle A A^{T}\left(Y-\widehat{\Pi}_{n} Y\right)+\widehat{\Pi}_{n} Y,\left(A A^{T}\right)^{\dagger 3} Y\right\rangle} \\
& =\frac{\left\langle A\left(A^{T} A\right)^{-3} \widehat{X}, A \widehat{X}+\widehat{\eta}\right\rangle}{\left\langle A\left(A^{T} A\right)^{-3}\left(\widehat{X}+A^{T} \widehat{\eta}\right), A \widehat{X}+\widehat{\eta}\right\rangle} \\
& =1-\frac{\left\langle\left(A A^{T}\right)^{\dagger 2} \widehat{\eta}, A \widehat{X}+\widehat{\eta}\right\rangle}{\left\langle A\left(A^{T} A\right)^{-3}\left(\widehat{X}+A^{T} \widehat{\eta}\right), A \widehat{X}+\widehat{\eta}\right\rangle} \\
& =\frac{\sum_{i=1}^{d} \sigma_{i}^{-5} \widehat{\alpha}_{i}}{\sum_{i=1}^{d} \sigma_{i}^{-5} \widehat{\alpha}_{i}\left(\sigma_{i} \widehat{\nu}_{i} \widehat{\xi}_{i}^{-1}+1\right)} \\
& =1-\frac{\sum_{i=1}^{d} \sigma_{i}^{-4} \widehat{\alpha}_{i} \widehat{\nu}_{i}}{\sum_{i=1}^{d} \sigma_{i}^{-5} \widehat{\alpha}_{i}\left(\sigma_{i} \widehat{\nu}_{i} \widehat{\xi}_{i}^{-1}+1\right)} .
\end{aligned}
$$

In Figure 11, we present the comparison between optimal parameters $t^{*}$ and their approximations $\widehat{t}_{n}^{\text {in }}$. Despite the fact that the gap between $\sigma_{d}$ and $1-t^{*}$ is not as large as requested in (42), the agreement between $t^{*}$ and $\widehat{t}_{n}^{\text {in }}$ keeps rather satisfactory. In Figure 12, we report the empirical distributions of the parameters, showing essentially their agreement. 


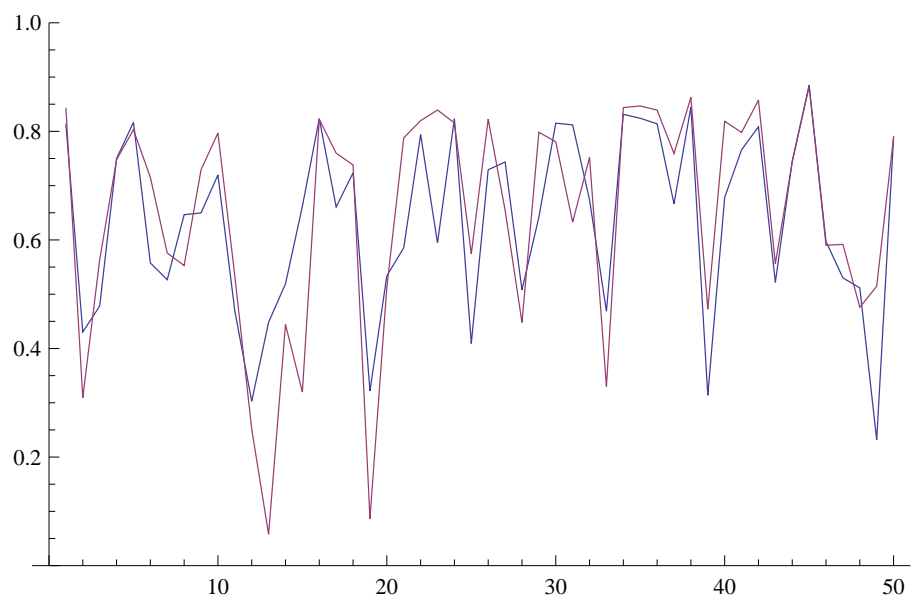

Figure 11: Optimal parameters $t^{*}$ and corresponding approximations $\widehat{t}_{n}(n=1000)$ for 50 different data $Y=A X+\eta$ for $X$ and $\eta$ generated as for the experiment of Figure 2 . Here we considered a noise level $\sigma=0.006$, so that the optimal parameter $t^{*}$ can be very close to 0.5 and the minimal singular value of $A$ is $\sigma_{d} \approx 0.7$.
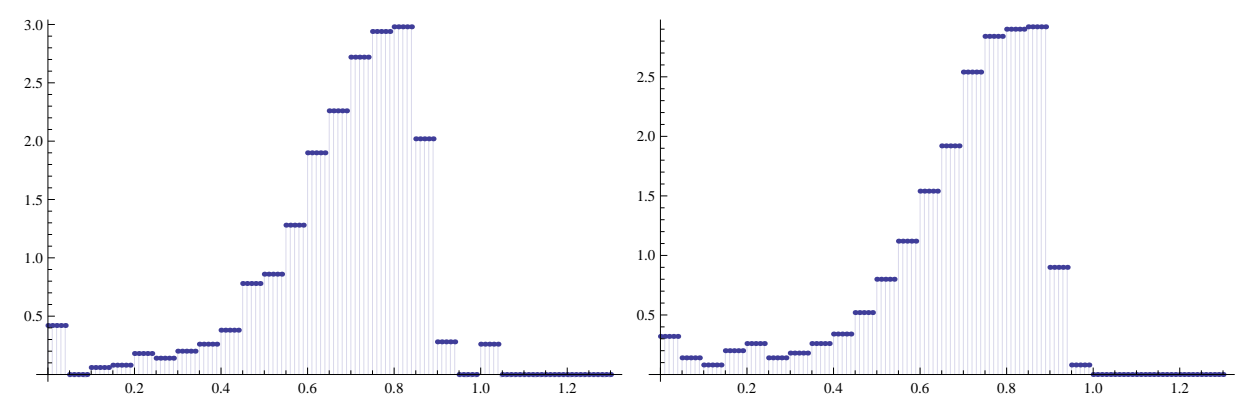

Figure 12: Empirical distribution of the optimal parameters $t^{*}$ (left) and the corresponding empirical distribution of the approximating parameters $t_{n}^{\text {in }}$ (right) for 1000 randomly generated data $Y=A X+\eta$ with the same higher noise level. The statistical accordance of the two parameters $t^{*}$ and $t_{n}^{\text {tin }}$ is shown.

\section{Conclusions and a glimps to future directions}

Motivated by the challenge of the regularization and parameter choice/learning relevant for many inverse problems in real-life, in this paper we presented a method to determine the parameter, based on the usage of a supervised machine learning framework. Under the assumption that the solution of the inverse problem is distributed sub-gaussianly over a small dimensional linear subspace $\mathcal{V}$ and the noise is also sub-gaussian, we provided a rigorous theoretical justification for the learning procedure of the function, which maps given noisy data into an optimal Tikhonov regularization parameter. We also presented and discussed explicit bounds for special cases and provided techniques for the practical implementation 
of the method.

Classical empirical computations of Tikhonov regularization parameters, for instance the discrepancy principle, see, e.g., (Bauer and Lukas, 2011; Engl et al., 1996), may be assuming the knowledge of the noise level $\sigma$ and use regularized Tikhonov solutions for different choices of $t$

$$
Z^{t}=t\left(t A^{T} A+(1-t) I\right)^{-1} A^{T} Y,
$$

to return some parameter $\widehat{t}_{\sigma}$ which ideally provides $\sigma$-optimal asymptotic behavior of $Z^{\widehat{t}_{\sigma}}$ for noise level $\sigma \rightarrow 0$. To be a bit more concrete, by using either bisection-type methods or by fixing a pretermined grid of points $\mathcal{T}=\left\{t^{(j)}: j=1,2, \ldots\right\}$, one searches some $t^{(\bar{j})} \in \mathcal{T}$ for which $\left\|A Z^{t^{(\bar{j})}}-Y\right\| \approx \sigma$ and set $\widehat{t}_{\sigma}:=t^{(\bar{j})}$. For each $t=t^{(j)}$ the computation of $Z^{t}$ has cost of order $\mathcal{O}\left(d^{2}\right)$ as soon as one has precomputed the SVD of $A$. Most of the empirical estimators require then the computation of $N_{i t}$ instances of $Z^{t}$ for a total of $\mathcal{O}\left(N_{i t} d^{2}\right)$ operations to return $\widehat{t}_{\sigma}$.

Our approach does not assume knowledge of $\sigma$ and it fits into the class of heuristic parameter choice rules, see (Kindermann, 2011); it requires first to have precomputed the empirical projection $\widehat{\Pi}_{n}$ by computation of one single $h$-truncated $\mathrm{SVD}^{2}$ of the empirical covariance matrix $\widehat{\Sigma}_{n}$ of dimension $m \times m$, where $m$ is assumed to be significantly smaller than $d$. In fact, the complexity of methods for computing a truncated SVD out of standard books is $\mathcal{O}\left(h \mathrm{~m}^{2}\right)$. When one uses randomized algorithms one could reduce the complexity even to $\mathcal{O}\left(\log (h) m^{2}\right)$, see (Halko et al., 2011). Then one needs to solve the optimization problem.

$$
\min _{t \in[0,1]}\left\|Z^{t}-\widehat{X}\right\|^{2}
$$

This optimization is equivalent to finding numerically roots in $(0,1)$ of the function $\widehat{H}_{n}(t)$, which can be performed in the general case and for $d>2$ only by iterative algorithms. They also require sequential evaluations of $Z^{t}$ of individual cost $\mathcal{O}\left(d^{2}\right)$, but they converge usually very fast and they are guaranteed by our results to return $Z^{\widehat{t}} \approx Z^{t^{*}}$. If we denote $N_{i t}^{\circ}$ the number of iterations needed for the root finding with appropriate accuracy, we obtain a total complexity of $\mathcal{O}\left(N_{i t}^{\circ} d^{2}\right)$. In the general case, our approach is going to be more advantageous with respect to classical methods if $N_{i t}^{\circ} \ll N_{i t}$. However, in the special case where the noise level is relatively small compared to the minimal positive singular value of $A$, e.g., $\sigma_{d} \gg \sigma$, then our arguments in Section 5 suggest that one can approximate the relevant root of $\widehat{H}_{n}(t)$ and hence $t^{*}$ with the smaller cost of $\mathcal{O}\left(d^{2}\right)$ and no need of several evaluations of $Z^{t}$. As a byproduct of our results we also showed that

$$
\widehat{X}=A^{\dagger} \widehat{\Pi}_{n} Y
$$

is also a good estimator and this one requires actually only the computation of $\widehat{\Pi}_{n}$ and then the execution of the operations in formula (44) of cost $\mathcal{O}(m d)$ (if we assumed the precomputation of the SVD of $A$ ).

2. One needs to compute $h$ singular values and singular vectors up to the first significant spectral gap. 
Our current efforts are devoted to the extension of the analysis to the case where the underlying space $\mathcal{V}$ is actually a smooth lower-dimensional nonlinear manifold. This extension will be realized by firstly approximating the nonlinear manifold locally on a proper decomposition by means of affine spaces as proposed in (Chen et al., 2013) and then applying our presented results on those local linear approximations.

Another interesting future direction consists of extending the approach to sets $\mathcal{V}$, unions of linear subspaces as in the case of solutions expressible sparsely the respect to certain dictionaries. In this situation, one would need to consider different regularization techniques and possibly non-convex non-smooth penalty quasi-norms.

For the sake of providing a first glimps on the feasibility of the latter possible extension, we consider below the problem of image denoising. In particular, as a simple example of the image denoising algorithm, we consider the wavelet shrinkage (Donoho and Johnstone, 1994): given a noisy image $Y=X+\sigma W$ (already expressed in wavelet coordinates), where $\sigma$ is the level of Gaussian noise, the denoised image is obtained by

$$
Z^{\alpha}=\mathbb{S}_{\alpha}(X)=\arg \min _{Z}\|Z-Y\|^{2}+2 \alpha\|Z\|_{\ell_{1}},
$$

where $\|\cdot\|_{\ell_{1}}$ denotes the $\ell_{1}$ norm, which promotes a sparse representation of the image with respect to a wavelet decomposition. Here, as earlier, we are interested in learning the highdimensional function mapping noisy images $X$ into their optimal shrinkage parameters, i.e., an optimal solution of $\left\|Z^{\alpha}-X\right\|^{2} \rightarrow \min _{\alpha}$.

Employing a properly modified version of the procedure described in this paper, we are obtaining very exciting and promising results, in particular that the optimal shrinkage parameter $\alpha$ essentially depends nonlinearly on very few (actually 1 or 2 ) linear evaluations of $Y$. This is not a new observation and it is a data-driven verification of the well-known results of (Donoho and Johnstone, 1994) and (Chambolle et al., 1998), establishing that the optimal parameter depends essentially on two meta-features of the noisy image, i.e., the noise level and its Besov regularity. In Figure 13 and Figure 14 we present the numerical results for wavelet shrinkage, which show that our approach chooses a nearly optimal parameter in terms of peak signal-to-noise ratio (PSNR) and visual quality of the denoising.

\section{Acknowledgments}

E. De Vito is a member of the Gruppo Nazionale per l'Analisi Matematica, la Probabilità e le loro Applicazioni (GNAMPA) of the Istituto Nazionale di Alta Matematica (INdAM). M. Fornasier acknowledges the financial support of the ERC-Starting Grant HDSPCONTR "High-Dimensional Sparse Optimal Control". V. Naumova acknowledges the support of project "Function-driven Data Learning in High Dimension" (FunDaHD) funded by the Research Council of Norway.

\section{Appendix A. Perturbation result for compact operators}

We recall the following perturbation result for compact operators in Hilbert spaces (Anselone, 1971) and (Zwald and Blanchard, 2006, Theorem 3), whose proof also holds without the assumption that the spectrum is simple (see Rosasco et al., 2010, Theorem 20). 

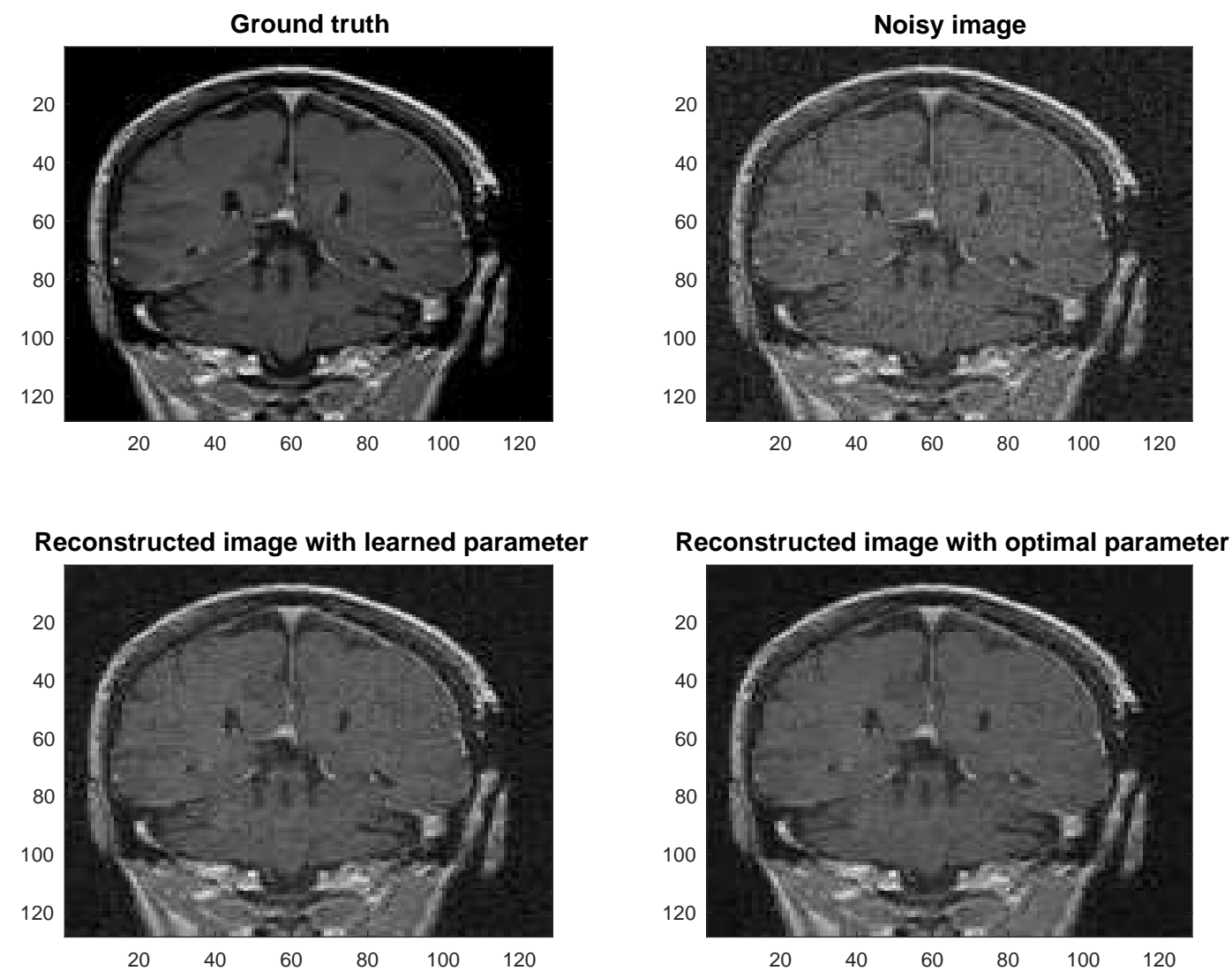

Figure 13: Numerical Experiments for Wavelet Shrinkage.

Proposition 15 Let $\mathcal{A}$ and $\mathcal{B}$ be two compact positive operators on a Hilbert space $\mathcal{H}$ and denote by $\left(\alpha_{j}\right)_{j=1}^{N}$ and $\left(\beta_{\ell}\right)_{\ell=1}^{L}$ the corresponding families of (distinct) strictly positive eigenvalues of $\mathcal{A}$ and $\mathcal{B}$ ordered in a decreasing way. For all $1 \leq j \leq N$, denote by $P_{j}$ (resp. $Q_{\ell}$ with $\left.1 \leq \ell \leq L\right)$ the projection onto the vector space spanned by the eigenvectors of $\mathcal{A}$ (resp. $\mathcal{B})$ whose eigenvalues are greater or equal than $\alpha_{j}$ (respect. $\left.\beta_{\ell}\right)$. Let $j \leq N$ such that $\|\mathcal{A}-\mathcal{B}\|<\frac{\alpha_{j}-\alpha_{j+1}}{4}$, then there exists $\ell \leq L$ so that

$$
\begin{aligned}
& \beta_{\ell+1}<\frac{\alpha_{j}+\alpha_{j+1}}{2}<\beta_{\ell} \\
& \left\|Q_{\ell}-P_{j}\right\| \leq \frac{2}{\alpha_{j}-\alpha_{j+1}}\|\mathcal{A}-\mathcal{B}\| \\
& \operatorname{dim} Q_{\ell} \mathcal{H}=\operatorname{dim} P_{j} \mathcal{H} .
\end{aligned}
$$

If $\mathcal{A}$ and $\mathcal{B}$ are Hilbert-Schmidt, the operator norm in the above bound can be replaced by the Hilbert-Schmidt norm. 


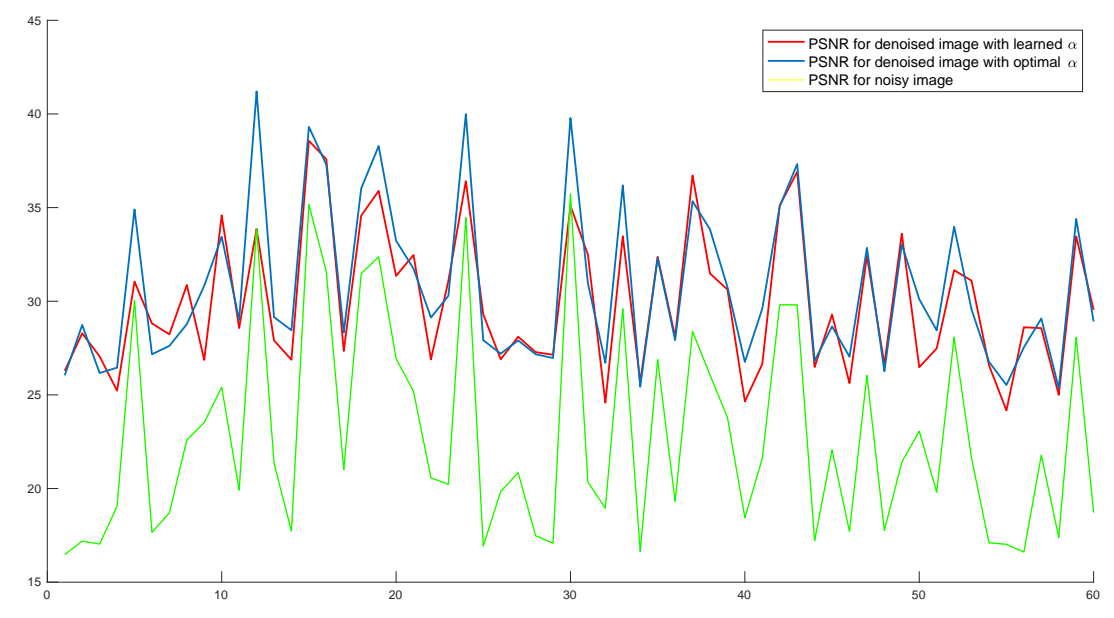

Figure 14: PSNR between the ground truth image and its noisy version (green line); the ground truth and its denoised version with the optimal parameter (blue line) and the learned parameter (red line). The results are presented for 60 random images.

In the above proposition, if $N$ or $M$ are finite, $\alpha_{N+1}=0$ or $\beta_{L+1}=0$. We note that, if the eigenvalues of $A$ or $B$ are not simple, in general $\ell \neq j$. However, the above result shows that, given $j=1, \ldots, N$ there exists a unique $\ell=1, \ldots, L$ such that $\operatorname{dim} Q_{\ell} \mathcal{H}=\operatorname{dim} P_{j} \mathcal{H}$ and $\beta_{\ell}$ is the smallest eigenvalue of $B$ greater than $\left(\alpha_{j}+\alpha_{j+1}\right) / 2$.

\section{Appendix B. Sub-gaussian vectors}

We recall some facts about sub-gaussian random vectors and we follow the presentation in (Vershynin, 2012), which provides the proofs of the main results in Lemma 5.5.

Proposition 16 Let $\xi$ be a sub-gaussian random vector in $\mathbb{R}^{d}$. Then, for all $\tau>0$ and $v \in \mathbb{R}^{d}$

$$
\mathbb{P}\left[|\langle\xi, v\rangle|>3\|\xi\|_{\psi_{2}}\|v\| \tau\right] \leq 2 \exp \left(-\tau^{2}\right)
$$

Under the further assumption that $\xi$ is centered, then

$$
\begin{aligned}
\mathbb{E}[\exp (\tau\langle\xi, v\rangle)] & \leq \exp \left(8 \tau^{2}\|\xi\|_{\psi_{2}}^{2}\right) \\
\mathbb{P}\left[\langle\xi, v\rangle>4 \sqrt{2}\|\xi\|_{\psi_{2}}\|v\| \tau\right] & \leq \exp \left(-\tau^{2}\right) .
\end{aligned}
$$

Proof We follow the idea in Vershynin (2012, Lemma 5.5) of explicitly computing the constants. By rescaling $\xi$ to $\xi /\|\xi\|_{\psi_{2}}$, we can assume that $\|\xi\|_{\psi_{2}}=1$. 
Let $c>0$ be a small constant to be fixed. Given, $v \in S^{d-1}$, set $\chi=\langle\xi, v\rangle$, which is a real sub-gaussian vector. By Markov inequality,

$$
\begin{aligned}
\mathbb{P}[|\chi|>\tau] & =\mathbb{P}\left[c|\chi|^{2}>c \tau^{2}\right]=\mathbb{P}\left[\exp \left(c|\chi|^{2}\right)>\exp \left(c \tau^{2}\right)\right] \\
& \leq \mathbb{E}\left[\exp \left(c|\chi|^{2}\right)\right] \mathrm{e}^{-c \tau^{2}}
\end{aligned}
$$

By (5), we get that $\mathbb{E}\left[|\chi|^{q}\right] \leq q^{q / 2}$, so that

$$
\mathbb{E}\left[\exp \left(c|\chi|^{2}\right)\right]=1+\sum_{k=1}^{+\infty} \frac{c^{k}}{k !} \mathbb{E}\left[|\chi|^{2 k}\right] \leq 1+\sum_{k=1}^{+\infty} \frac{(2 c k)^{k}}{k !} \leq 1+\frac{1}{\mathrm{e}} \sum_{k=1}^{+\infty}(2 c e)^{k}=1+\frac{2 c}{1-2 c \mathrm{e}},
$$

where we use the estimate $k ! \geq \mathrm{e}(k / \mathrm{e})^{k}$ for $k \geq 1$. Setting $c=1 / 9,1+\frac{2 c}{1-2 c e}<2$, so that

$$
\mathbb{P}[|\chi|>3 \tau] \leq 2 \exp \left(-\tau^{2}\right)
$$

so that (46a) is proven.

Assume now that $\mathbb{E}[\xi]=0$. By (5.8) in Vershynin (2012)

$$
\mathbb{E}\left[\exp \left(\frac{\tau}{\mathrm{e}} \chi\right)\right] \leq 1+\sum_{k=2}^{+\infty}\left(\frac{|\tau|}{\sqrt{k}}\right)^{k}
$$

and, by (5.9) in Vershynin (2012),

$$
\exp \left(\tau^{2} C^{2}\right) \geq 1+\sum_{h=1}^{+\infty}\left(\frac{C|\tau|}{\sqrt{h}}\right)^{2 h}
$$

If $|\tau| \leq 1$, fix an even $k \geq 2$ so that $k=2 h$ with $h \geq 1$. The $k$-th and $(k+1)$-th terms of the series (47) is

$$
\left(\frac{|\tau|}{\sqrt{k}}\right)^{k}+\left(\frac{|\tau|}{\sqrt{k+1}}\right)^{k+1} \leq 2\left(\frac{|\tau|}{\sqrt{k}}\right)^{k}=\frac{2}{C^{2 h} 2^{h}}\left(\frac{|\tau|}{\sqrt{2 h}}\right)^{2 h} \leq\left(\frac{C|\tau|}{\sqrt{k}}\right)^{k},
$$

where the right hand side is the $h$-th term of the series (48) and the last inequality holds true if $C \geq 1$. Under this assumption

$$
\mathbb{E}\left[\exp \left(\frac{\tau}{\mathrm{e}} \chi\right)\right] \leq \exp \left(\tau^{2} C^{2}\right) \quad|\tau| \leq 1
$$

If $|\tau| \geq 1$, fix an odd $k \geq 3$, so that $k=2 h-1$ with $h \geq 2$, then the $k$-th and $(k+1)$-th terms of the series (47) is

$$
\left(\frac{|\tau|}{\sqrt{k}}\right)^{k}+\left(\frac{|\tau|}{\sqrt{k+1}}\right)^{k+1} \leq 2\left(\frac{|\tau|}{\sqrt{k}}\right)^{k+1}=\left(\frac{\sqrt{2} h}{C^{2}(2 h-1)}\right)^{h}\left(\frac{|\tau|}{\sqrt{h}}\right)^{2 h} \leq\left(\frac{|\tau|}{\sqrt{h}}\right)^{2 h}
$$

where the right hand side is the $h$-th term of the series (48) and the inequality holds true provided that

$$
C^{2} \geq \sup _{h \geq 2} \frac{\sqrt{2} h}{(2 h-1)}=\frac{2 \sqrt{2}}{3},
$$


which holds true with the choice $C=1$. Furthermore, the term of (47) with $k=2$ is clearly bounded by term of (48) with $h=1$, so that we get

$$
\mathbb{E}\left[\exp \left(\frac{\tau}{\mathrm{e}} \chi\right)\right] \leq \exp \left(\tau^{2}\right) \quad|\tau| \geq 1
$$

Together with bound (49) with $C=1$, the abound bound gives (46b) since $e^{2}<8$.

Finally, reasoning as in the proof of (46a) and by using (46b)

$$
\begin{aligned}
\mathbb{P}[\chi>\tau] & =P[\exp (c \chi)>\exp (c \tau)] \\
& \leq \mathbb{E}[\exp (c \chi)] \mathrm{e}^{-c \tau} \\
& \leq \exp \left(8 c^{2}-c \tau\right)
\end{aligned}
$$

which takes the minimum at $c=\tau / 16$. Hence,

$$
\mathbb{P}[\chi>\tau]=\exp \left(-\frac{\tau^{2}}{32}\right)
$$

Remark 17 Both (46a) and (46b) (for a suitable constants instead of $\|\xi\|_{\gamma_{2}}$ ) are sufficient conditions for sub-gaussianity and (46b) implies that $\mathbb{E}[\xi]=0$, see (Vershynin, 201\%, Lemma 5.5).

The following proposition bounds the Euclidean norm of a sub-gaussian vector. The proof is standard and essentially based on the results in Vershynin (2012), but we were not able to find the precise reference. The centered case is done in Rigolet (2015).

Proposition 18 Let $\xi$ a sub-gaussian random vector in $\mathbb{R}^{d}$. Given $\tau>0$ with probability greater than $1-2 \mathrm{e}^{-\tau^{2}}$

$$
\|\xi\| \leq 3\|\xi\|_{\psi_{2}}(\sqrt{7 d}+2 \tau) \leq 9\|\xi\|_{\psi_{2}}(\sqrt{d}+\tau) .
$$

If $\mathbb{E}[\xi]=0$, then with probability greater than $1-\mathrm{e}^{-\tau^{2}}$

$$
\|\xi\| \leq 8\|\xi\|_{\psi_{2}}\left(\sqrt{\frac{7}{2}} d+\sqrt{2} \tau\right) \leq 16\|\xi\|_{\psi_{2}}(\sqrt{d}+\tau) .
$$

Proof As usual we assume that $\|\xi\|_{\psi_{2}}=1$. Let $\mathcal{N}$ be a $1 / 2$-net of $S^{d-1}$. Lemmas 5.2 and 5.3 in Vershynin (2012) give

$$
\|\xi\| \leq 2 \max _{v \in \mathcal{N}}\langle\xi, v\rangle \quad|\mathcal{N}| \leq 5^{d} .
$$

Fixed $v \in \mathcal{N}$, (46a) gives that

$$
\mathbb{P}[|\langle\xi, v\rangle|>3 t] \leq 2 \exp \left(-t^{2}\right) .
$$


By union bound

$$
\mathbb{P}[\|\xi\|>6 t] \leq \mathbb{P}\left[\max _{v \in \mathcal{N}}|\langle\xi, v\rangle|>3 t\right] \leq 2|\mathcal{N}| \exp \left(-t^{2}\right) \leq 2 \exp \left(d \ln 5-t^{2}\right)
$$

Bound (50) follows with the choice $t=\tau+\sqrt{7 d / 4}$ taking into account that $t^{2}-d \ln 5>\tau^{2}$ since $\ln 5<7 / 4$.

Assume that $\xi$ is centered and use (46c) instead of (46a). Then

$$
\mathbb{P}[\|\xi\|>8 \sqrt{2} t] \leq \mathbb{P}\left[\max _{v \in \mathcal{N}}|\langle\xi, v\rangle|>4 \sqrt{2} t\right] \leq|\mathcal{N}| \exp \left(-t^{2}\right) \leq \exp \left(d \ln 5-t^{2}\right) .
$$

As above, the choice $t=\tau+\sqrt{7 d / 4}$ provides the bound (51).

Remark 19 Compare with Theorem 1.19 in Rigolet (2015), noting that by (46b) the parameter $\sigma$ in Definition 1.2 of Rigolet (2015) is bounded by $4\|\xi\|_{\psi_{2}}$.

The following result is a concentration inequality for the second momentum of subgaussian random vector, see Theorem 5.39 and Remark 5.40 in Vershynin (2012) and footnote 20 .

Theorem 20 Let $\xi \in \mathbb{R}^{d}$ be a sub-gaussian vector random vector in $\mathbb{R}^{d}$. Given a family $\xi_{1}, \ldots, \xi_{n}$ of random vectors independent and identically distributed as $\xi$, then for $\tau>0$

$$
\mathbb{P}\left[\left\|\frac{1}{n} \sum_{i=1}^{n} \xi_{i} \otimes \xi_{i}-\mathbb{E}[\xi \otimes \xi]\right\|>\max \left\{\delta, \delta^{2}\right\}\right] \leq 2 \mathrm{e}^{-\tau^{2}}
$$

where

$$
\delta=C_{\xi}\left(\sqrt{\frac{d}{n}}+\frac{\tau}{\sqrt{n}}\right),
$$

and $C_{\xi}$ is a constant depending only on the sub-gaussian norm $\|\xi\|_{\psi_{2}}$.

\section{References}

William K. Allard, Guangliang Chen, and Mauro Maggioni. Multi-scale geometric methods for data sets. II: Geometric multi-resolution analysis. Appl. Comput. Harmon. Anal., 32 (3):435-462, 2012. ISSN 1063-5203. doi: 10.1016/j.acha.2011.08.001.

Philip M. Anselone. Collectively compact operator approximation theory and applications to integral equations. Prentice-Hall Inc., Englewood Cliffs, N. J., 1971.

Frank Bauer and Mark A. Lukas. Comparing parameter choice methods for regularization of ill-posed problems. Math. Comput. Simul., 81(9):1795-1841, 2011.

Andrea Braides. Gamma-convergence for beginners. Lecture notes. Available on line http://www.mat.uniroma2.it/ braides/0001/dotting.html, 2001. 
Antonin Chambolle, Ronald DeVore, Nam-yong Lee, and Bradley Lucier. Nonlinear wavelet image processing: variational problems, compression, and noise removal through wavelet shrinkage. IEEE Trans. Image Process., 7(3):319-335, 1998.

Guangliang Chen, Anna V. Little, and Mauro Maggioni. Multi-resolution geometric analysis for data in high dimensions. In Excursions in harmonic analysis. Volume 1, pages 259285. New York, NY: Birkhäuser/Springer, 2013.

David Donoho and Iain Johnstone. Ideal spatial adaptation by wavelet shrinkage. Biometrika, 81:425-55, 1994.

Heinz W. Engl, Martin Hanke, and Andreas Neubauer. Regularization of inverse problems. Dordrecht: Kluwer Academic Publishers, 1996.

N. Halko, P.G. Martinsson, and J.A. Tropp. Finding structure with randomness: probabilistic algorithms for constructing approximate matrix decompositions. SIAM Rev., 53 (2):217-288, 2011. ISSN 0036-1445; 1095-7200/e. doi: 10.1137/090771806.

Stefan Kindermann. Convergence analysis of minimization-based noise level-free parameter choice rules for linear ill-posed problems. ETNA, Electron. Trans. Numer. Anal., 38: 233-257, 2011. ISSN 1068-9613/e.

Gitta Kutyniok and Demetrio Labate, editors. Shearlets. Multiscale analysis for multivariate data. Boston, MA: Birkhäuser, 2012.

Anna V. Little, Mauro Maggioni, and Lorenzo Rosasco. Multiscale geometric methods for data sets. I: Multiscale SVD, noise and curvature. Appl. Comput. Harmon. Anal., 43(3): 504-567, 2017. ISSN 1063-5203. doi: 10.1016/j.acha.2015.09.009.

Stéphane Mallat. A wavelet tour of signal processing. The sparse way. 3rd ed. Amsterdam: Elsevier/Academic Press, 3rd ed. edition, 2009. ISBN 978-0-12-374370-1/hbk.

Erich Novak and Henryk Woźniakowski. Optimal order of convergence and (in)tractability of multivariate approximation of smooth functions. Constr. Approx., 30(3):457-473, 2009.

Philippe Rigolet. 18.S997: High dimensional statistics. Lecture notes. Available on line http://www-math.mit.edu/ rigollet/PDFs/RigNotes15.pdf, 2015.

Lorenzo Rosasco, Mikhail Belkin, and Ernesto De Vito. On learning with integral operators. J. Mach. Learn. Res., 11:905-934, 2010.

Mark Rudelson and Roman Vershynin. Hanson-Wright inequality and sub-Gaussian concentration. Electron. Commun. Probab., 18(82):1-9, 2013.

Roman Vershynin. Introduction to the non-asymptotic analysis of random matrices. In Compressed sensing, pages 210-268. Cambridge Univ. Press, Cambridge, 2012.

Laurent Zwald and Gilles Blanchard. On the convergence of eigenspaces in kernel principal component analysis. In Y. Weiss, B. Schölkopf, and J. Platt, editors, Advances in Neural Information Processing Systems 18, pages 1649-1656. MIT Press, Cambridge, MA, 2006. 